\title{
ON THE EXPRESSIVE POWER OF 2-STACK VISIBLY PUSHDOWN AUTOMATA
}

\author{
BENEDIKT BOLLIG
}

LSV, ENS Cachan, CNRS - 61, avenue du Président Wilson, 94235 Cachan Cedex, France e-mail address: bollig@lsv.ens-cachan.fr

\begin{abstract}
Visibly pushdown automata are input-driven pushdown automata that recognize some non-regular context-free languages while preserving the nice closure and decidability properties of finite automata. Visibly pushdown automata with multiple stacks have been considered recently by La Torre, Madhusudan, and Parlato, who exploit the concept of visibility further to obtain a rich automata class that can even express properties beyond the class of context-free languages. At the same time, their automata are closed under boolean operations, have a decidable emptiness and inclusion problem, and enjoy a logical characterization in terms of a monadic second-order logic over words with an additional nesting structure. These results require a restricted version of visibly pushdown automata with multiple stacks whose behavior can be split up into a fixed number of phases.

In this paper, we consider 2-stack visibly pushdown automata (i.e., visibly pushdown automata with two stacks) in their unrestricted form. We show that they are expressively equivalent to the existential fragment of monadic second-order logic. Furthermore, it turns out that monadic second-order quantifier alternation forms an infinite hierarchy wrt. words with multiple nestings. Combining these results, we conclude that 2 -stack visibly pushdown automata are not closed under complementation.

Finally, we discuss the expressive power of Büchi 2-stack visibly pushdown automata running on infinite (nested) words. Extending the logic by an infinity quantifier, we can likewise establish equivalence to existential monadic second-order logic.
\end{abstract}

\section{INTRODUCTION}

The notion of a regular word language has ever played an important rôle in computer science, as it constitutes a robust concept that enjoys manifold representations in terms of finite automata, regular expressions, monadic second-order logic, etc. Generalizing regular languages towards richer classes and more expressive formalisms is often accompanied by the loss of robustness and decidability properties. It is, for example, well-known that the class of context-free languages, represented by pushdown automata, is not closed under complementation and that universality, equivalence, and inclusion are undecidable problems $[12]$.

1998 ACM Subject Classification: F.4.3.

Key words and phrases: visibly pushdown automata, multiple stacks, nested words, monadic second-order logic. 
Visibly pushdown languages have been introduced by Alur and Madhusudan to overcome this deficiency while subsuming many interesting and useful context-free properties [1]. Visibly pushdown languages are represented by special pushdown automata whose stack operations are driven by the input. More precisely, the underlying alphabet of possible actions is partitioned into (1) call, (2) return, and (3) internal actions, which, when reading an action, indicates if (1) a stack symbol is pushed on the stack, (2) a stack symbol is read and popped from the stack, or (3) the stack is not touched at all, respectively. Such a partition gives rise to a call-return alphabet. Though this limits the expressive power of pushdown automata, the such defined class of visibly pushdown languages is rich enough to model various interesting non-regular properties for program analysis. Even more, this class preserves some important closure properties of regular languages, such as the closure under boolean operations, and it exhibits decidable problems, such as inclusion, that are undecidable in the context of general pushdown automata. Last but not least, the visibly pushdown languages are captured by a monadic second-order logic that makes use of a binary nesting predicate. Such a logic is suitable in the context of visibility, as the nesting structure of a word is uniquely determined, regardless of a particular run of the pushdown automaton. The logical characterization smoothly extends the classical theory of regular languages $[7,10]$. For context-free languages, quantification over matchings, which are not implicitly given when we do not have visibility, is necessary to obtain a logical characterization [15].

Visibly pushdown automata with multiple stacks have been considered recently and independently by La Torre, Madhusudan, and Parlato [13], as well as Carotenuto, Murano, and Peron [8]. The aim of these papers is to exploit the concept of visibility further to obtain even richer classes of non-regular languages while preserving important closure properties and decidability of verification-related problems such as emptiness and inclusion.

In [13], the authors consider visibly pushdown automata with arbitrarily many stacks. To retain the nice properties of visibly pushdown automata with only one stack, the idea is to restrict the domain, i.e., the possible inputs, to those words that can be divided into at most $k$ phases for a predefined $k$. In every phase, pop actions correspond to one and the same stack. These restricted visibly pushdown automata have a decidable emptiness problem, which is shown by a reduction to the emptiness problem for finite tree automata, and are closed under union, intersection, and complementation (wrt. the domain of $k$-phase words). Moreover, a word language is recognizable if, and only if, it can be defined in monadic second-order logic where the usual logic over words is expanded by a matching predicate that matches a push with its corresponding pop event. As mentioned above, such a matching is unique wrt. the underlying call-return alphabet. The only negative result in this regard is that multi-stack visibly pushdown automata cannot be determinized.

The paper [8] considers visibly pushdown automata with two stacks and call-return alphabets that appear more general than those of [13]: Any stack is associated with a partition of one and the same alphabet into call, return, and local transitions so that an action might be both a call action for the first stack and, at the same time, a return action for the second. In this way, both stacks can be worked on simultaneously. Note that, if we restrict to the alphabets of [13] where the stack alphabets are disjoint, the models from [8] and [13] coincide. Carotenuto et al. show that the emptiness problem of their model is undecidable. Their approach to gain decidability is to exclude simultaneous pop operations by introducing an ordering constraint on stacks, which is inspired by [6] (see also [3]). More precisely, a pop operation on the second stack is only possible if the first stack is empty. 
Under these restrictions, the emptiness problem turns out to be decidable in polynomial time (note that the number of stacks is fixed) 1

In this paper, we consider 2-stack visibly pushdown automata (i.e., visibly pushdown automata with two stacks) where each action is exclusive to one of the stacks, unless we deal with an internal action, which does not affect the stacks at all. Thus, we adopt the model of [13], though we have to restrict to two stacks for our main results. One of these results states that the corresponding language class is precisely characterized by the existential fragment of monadic second-order logic where a first-order kernel is preceded by a block of existentially quantified second-order variables. In a second step, we show that the full monadic second-order logic is strictly more expressive than its existential fragment so that we conclude that 2 -stack visibly pushdown automata are not closed under complementation. Note that our model has an undecidable emptiness problem, as can be easily seen.

The key technique in our proofs is to consider words over call-return alphabets as relational structures, called nested words [2]. Nested words augment ordinary words with a nesting relation that, as the logical atomic predicate mentioned above, relates push with corresponding pop events. More precisely, we consider a nested word to be a graph whose nodes are labeled with actions and are related in terms of a matching and an immediatepredecessor relation. We thus deal with structures of bounded degree: every node has at most two incoming edges (one from the immediate predecessor and one from a push event if we deal with a pop event operating on the non-empty stack) and, similarly, at most two outgoing edges. As there is a one-to-one correspondence between words and their nested counterpart, we may consider nested-word automata [2], which are equivalent to visibly pushdown automata but operate on the enriched word structures. There have been several notions of automata on graphs and partial orders $[18,19]$ that are similar to nested-word automata and have one idea in common: the state that is taken after executing some event depends on the states that have been visited in neighboring events. Such defined automata may likewise operate on models for concurrent-systems executions such as Mazurkiewicz traces [9] and message sequence charts [5]. In the framework of nested-word automata, to determine the state after executing a pop operation, we therefore have to consider both the state of the immediate-predecessor position and the state that had been reached after the execution of the corresponding push event. To obtain a logical characterization of nestedword automata over two stacks, we adopt a technique from [5]: for a natural number $r$, we compute a nested-word automaton $\mathcal{B}_{r}$ that computes the sphere of radius $r$ around any event $i$, i.e., the restriction of the input word to those events that have distance at most $r$ from $i$. Once we have this automaton, we can apply Hanf's Theorem, which states that satisfaction of a given first-order formula depends on the number of these local spheres counted up to a threshold that depends on the quantifier-nesting depth of the formula [11]. This finally leads us to a logical characterization of 2-stack visibly pushdown automata in terms of existential monadic second-order logic. Note that our construction of $\mathcal{B}_{r}$ is close to the nontrivial technique applied in [5]. In the context of nested words, however, the correctness proof is more complicated. The fact that we deal with two stacks only is crucial, and the construction fails as soon as a third stack comes into play.

\footnotetext{
${ }^{1}$ In [8], the authors argue that 2-stack visibly pushdown automata without restriction are closed under complementation, but their proof makes use of the incorrect assumption that these automata are determinizable. In fact, 2-stack visibly pushdown automata can in general not be determinized [13]. In the present paper, we show that 2 -stack visibly pushdown automata are actually not closed under complementation.
} 
Then, we exploit the concept of nested words to show that full monadic second-order logic is more expressive than its existential fragment. This is done by a first-order interpretation of nested words over two stacks into grids, for which the analogous result has been known [17].

An extension of Hanf's Theorem has been established to cope with infinite structures [4]. This allows us to apply the automaton $\mathcal{B}_{r}$ to also obtain a logical characterization of the canonical extension of 2-stack visibly pushdown automata towards Büchi automata running on infinite words.

Outline of the paper. In Section 2, we introduce multi-stack visibly pushdown automata, running on words, as well as multi-stack nested-word automata, which operate on nested words. We establish expressive equivalence of these two models. Section 3 recalls monadic second-order logic over relational structures and, in particular, nested words. There, we also state Hanf's Theorem, which provides a normal form of first-order definable properties in terms of spheres. The construction of the sphere automaton $\mathcal{B}_{r}$, which is, to some extent, the core contribution of this paper, is the subject of Section 4.2. By means of this automaton, we can show expressive equivalence of 2-stack visibly pushdown automata and existential monadic second-order logic (Section 4.1). Section 5 establishes the gap between this fragment and the full logic, from which we conclude that 2-stack visibly pushdown automata cannot be complemented in general. By slightly modifying our logic, we obtain, in Section 6, a characterization of Büchi 2-stack visibly pushdown automata, running on infinite words. We conclude with Section 7 stating some related open problems.

\section{Multi-Stack Visibly Pushdown Automata}

The set $\{0,1,2, \ldots\}$ of natural numbers is denoted by $\mathbb{N}$, the set $\{1,2, \ldots\}$ of positive natural numbers by $\mathbb{N}_{+}$. We call any finite set an alphabet. For a set $\Sigma$, we denote by $\Sigma^{*}$, $\Sigma^{+}$, and $\Sigma^{\omega}$ the sets of finite, nonempty finite, and infinite strings over $\Sigma$, respectively 2 The empty string is denoted by $\varepsilon$. For a natural number $n \in \mathbb{N}$, we let $[n]$ stand for the set $\{1, \ldots, n\}$ (i.e., [0] is the empty set). In this paper, we will identify isomorphic structures and we use $\cong$ to denote isomorphism.

Let $K \geq 1$ be a positive natural number. A ( $K$-stack) call-return alphabet is a collection $\left\langle\left\{\left(\Sigma_{c}^{s}, \Sigma_{r}^{s}\right)\right\}_{s \in[K]}, \Sigma_{\text {int }}\right\rangle$ of pairwise disjoint alphabets. Intuitively, $\Sigma_{c}^{s}$ contains the actions that call the stack $s, \Sigma_{r}^{s}$ is the set of returns of stack $s$, and $\Sigma_{i n t}$ is a set of internal actions, which do not involve any stack operation.

We fix $K \geq 1$ and a $K$-stack call-return alphabet $\widetilde{\Sigma}=\left\langle\left\{\left(\Sigma_{c}^{s}, \Sigma_{r}^{s}\right)\right\}_{s \in[K]}, \Sigma_{\text {int }}\right\rangle$. Moreover, we set $\Sigma_{c}=\bigcup_{s \in[K]} \Sigma_{c}^{s}, \Sigma_{r}=\bigcup_{s \in[K]} \Sigma_{r}^{s}$, and $\Sigma=\Sigma_{c} \cup \Sigma_{r} \cup \Sigma_{\text {int }}$.

\subsection{Multi-Stack Visibly Pushdown Automata.}

Definition 2.1. A multi-stack visibly pushdown automaton (MvPA) over $\widetilde{\Sigma}$ is a tuple $\mathcal{A}=$ $\left(Q, \Gamma, \delta, Q_{I}, F\right)$ where

- $Q$ is its finite set of states,

- $Q_{I} \subseteq Q$ is the set of initial states,

- $F \subseteq Q$ is the set of final states,

\footnotetext{
${ }^{2}$ From now on, to avoid confusion with nested words, we use the term "string" rather than "word" if we deal with elements from $\Sigma^{*} \cup \Sigma^{\omega}$.
} 
- $\Gamma$ is the finite stack alphabet containing a special symbol $\perp$ that will represent the empty stack, and

- $\delta$ provides the transitions in terms of a triple $\left\langle\delta_{c}, \delta_{r}, \delta_{i n t}\right\rangle$ with

$$
\begin{aligned}
\delta_{c} & \subseteq Q \times \Sigma_{c} \times(\Gamma \backslash\{\perp\}) \times Q, \\
\delta_{r} & \subseteq Q \times \Sigma_{r} \times \Gamma \times Q, \text { and } \\
\delta_{i n t} & \subseteq Q \times \Sigma_{i n t} \times Q .
\end{aligned}
$$

A 2-stack visibly pushdown automaton (2VPA) is an MvPA that is defined over a 2-stack alphabet (i.e., $K=2$ ).

A transition $\left(q, a, A, q^{\prime}\right) \in \delta_{c}$, say with $a \in \Sigma_{c}^{s}$, is a push transition meaning that, being in state $q$, the automaton can read $a$, push the symbol $A \in \Gamma \backslash\{\perp\}$ onto the $s$-th stack, and go over to state $q^{\prime}$. A transition $\left(q, a, A, q^{\prime}\right) \in \delta_{r}$, say with $a \in \Sigma_{r}^{s}$, allows us to pop $A \neq \perp$ from the $s$-th stack when reading $a$, while the control changes from state $q$ to state $q^{\prime}$. If, however, $A=\perp$, then the stack is not touched, i.e., $\perp$ is never popped. Finally, a transition $\left(q, a, q^{\prime}\right) \in \delta_{\text {int }}$ is applied when reading internal actions $a \in \Sigma_{\text {int }}$. They do not involve any stack operation and, actually, do not even allow us to read from the stack.

Let us formalize the behavior of the MvpA $\mathcal{A}$. A stack contents is a nonempty finite sequence from Cont $=(\Gamma \backslash\{\perp\})^{*} \cdot\{\perp\}$. The leftmost symbol is thus the top symbol of the stack contents. A configuration of $\mathcal{A}$ consists of a state and a stack contents for every stack. Hence, it is an element of $Q \times C o n t^{[K]}$. Consider a string $w=a_{1} \ldots a_{n} \in \Sigma^{+}$. A run of $\mathcal{A}$ on $w$ is a sequence $\rho=\left(q_{0}, \sigma_{0}^{1}, \ldots, \sigma_{0}^{K}\right) \ldots\left(q_{n}, \sigma_{n}^{1}, \ldots, \sigma_{n}^{K}\right) \in\left(Q \times \text { Cont }^{[K]}\right)^{+}$such that $q_{0} \in Q_{I}, \sigma_{0}^{s}=\perp$ for each stack $s \in[K]$, and, for all $i \in\{1, \ldots, n\}$, the following hold:

[Push]: If $a_{i} \in \Sigma_{c}^{s}$ for $s \in[K]$, then there is a stack symbol $A \in \Gamma \backslash\{\perp\}$ such that $\left(q_{i-1}, a_{i}, A, q_{i}\right) \in \delta_{c}, \sigma_{i}^{s}=A \cdot \sigma_{i-1}^{s}$, and $\sigma_{i}^{s^{\prime}}=\sigma_{i-1}^{s^{\prime}}$ for every $s^{\prime} \in[K] \backslash\{s\}$.

[Pop]: If $a_{i} \in \Sigma_{r}^{s}$ for $s \in[K]$, then there is a stack symbol $A \in \Gamma$ such that $\left(q_{i-1}, a_{i}, A, q_{i}\right) \in$ $\delta_{r}, \sigma_{i}^{s^{\prime}}=\sigma_{i-1}^{s^{\prime}}$ for every $s^{\prime} \in[K] \backslash\{s\}$, and either $A \neq \perp$ and $\sigma_{i-1}^{s}=A \cdot \sigma_{i}^{s}$, or $A=\perp$ and $\sigma_{i-1}^{s}=\sigma_{i}^{s}=\perp$.

[Internal]: If $a_{i} \in \Sigma_{\text {int }}$, then $\left(q_{i-1}, a_{i}, q_{i}\right) \in \delta_{\text {int }}$, and $\sigma_{i}^{s}=\sigma_{i-1}^{s}$ for every $s \in[K]$.

The run $\rho$ is accepting if $q_{n} \in F$. A string $w \in \Sigma^{+}$is accepted by $\mathcal{A}$ if there is an accepting run of $\mathcal{A}$ on $w$. The set of accepted strings forms the (string) language of $\mathcal{A}$, which is a subset of $\Sigma^{+}$and denoted by $L(\mathcal{A}){ }^{3}$

Example 2.2. There is no MvPA that recognizes the context-sensitive language $\left\{a^{n} b^{n} c^{n} \mid\right.$ $n \geq 1$, no matter which call-return alphabet we chose. Note that, however, with the more general notion of a call-return alphabet from [8], it is possible to recognize this language by means of two stacks. Now consider the 2-stack call-return alphabet $\widetilde{\Sigma}$ given by $\Sigma_{c}^{1}=\{a\}$, $\Sigma_{r}^{1}=\{\bar{a}\}, \Sigma_{c}^{2}=\{b\}, \Sigma_{r}^{2}=\{\bar{b}\}$, and $\Sigma_{\text {int }}=\emptyset$. The language $L=\left\{(a b)^{n} \bar{a}^{n+1} \bar{b}^{n+1} \mid n \geq 1\right\}$ can be recognized by some $2 \mathrm{VPA}$ over $\widetilde{\Sigma}$, even by the restricted model of 2-phase $2 \mathrm{VPA}$ from [13], as every word from $L$ can be split into at most two return phases. In the following, we define a 2 VPA $\mathcal{A}=\left(\left\{q_{0}, \ldots, q_{4}\right\},\{\$, \perp\}, \delta,\left\{q_{0}\right\},\left\{q_{0}\right\}\right)$ over $\widetilde{\Sigma}$ such that $L(\mathcal{A})=L^{+}$, which is no longer divisible into a bounded number of return phases. The transition relation $\delta$ is

\footnotetext{
${ }^{3}$ To simplify the presentation, the empty word $\varepsilon$ is excluded from the domain.
} 
given as follows (a graphical illustration is provided in Figure 1):

$$
\begin{aligned}
& \delta_{c}:\left(q_{0}, a, \$, q_{2}\right) \quad \delta_{r}: \quad\left(q_{3}, \bar{a}, \$, q_{3}\right) \\
& \left(q_{2}, b, \$, q_{1}\right) \quad\left(q_{3}, \bar{a}, \perp, q_{4}\right) \\
& \left(q_{1}, a, \$, q_{2}\right) \quad\left(q_{4}, \bar{b}, \$, q_{4}\right) \\
& \left(q_{2}, b, \$, q_{3}\right) \quad\left(q_{4}, \bar{b}, \perp, q_{0}\right)
\end{aligned}
$$

The idea is that the finite-state control ensures that an input word matches the regular expression $\left((a b)^{+} \bar{a}^{+} \bar{b}^{+}\right)^{+}$. To guarantee that, in any iteration, the number of $a$ is by one less than the number of $\bar{a}$, any push action $a$ stores a stack symbol $\$$ in stack 1 , which can then be removed by the corresponding pop action $\bar{a}$ unless the symbol $\perp$ is discovered. We do the same for $b$ and $\bar{b}$ on stack 2 .

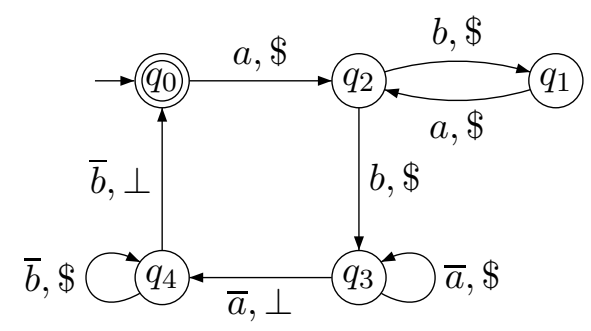

Figure 1: A 2VPA

2.2. Nested Words and Multi-Stack Nested-Word Automata. We will now see how strings over symbols from the call-return alphabet $\widetilde{\Sigma}$ can be represented by relational structures. Basically, to a string, we add a binary predicate that combines push with corresponding pop events. Let $s \in[K]$. A string $w \in \Sigma^{*}$ is called $s$-well formed if it is generated by the context-free grammar

$$
A::=a A b|A A| \varepsilon \mid c
$$

where $a \in \Sigma_{c}^{s}, b \in \Sigma_{r}^{s}$, and $c \in \Sigma \backslash\left(\Sigma_{c}^{s} \cup \Sigma_{r}^{s}\right)$.

Definition 2.3. A nested word over $\widetilde{\Sigma}$ is a structure $([n], \lessdot, \mu, \lambda)$ where $n \in \mathbb{N}_{+}$(we call the elements from $[n]$ positions, nodes, or events $), \lessdot=\{(i, i+1) \mid i \in[n-1]\}, \lambda:[n] \rightarrow \Sigma$, and $\mu=\bigcup_{s \in[K]} \mu^{s} \subseteq[n] \times[n]$ where, for every $s \in[K]$ and $(i, j) \in[n] \times[n],(i, j) \in \mu^{s}$ iff $i<j, \lambda(i) \in \Sigma_{c}^{s}, \lambda(j) \in \Sigma_{r}^{s}$, and $\lambda(i+1) \ldots \lambda(j-1)$ is $s$-well formed.

The set of nested words over $\widetilde{\Sigma}$ is denoted by $\mathbb{N W W}(\widetilde{\Sigma})$.

Figure 2 depicts a nested word over a 2-stack call-return alphabet. Throughout the paper, we take advantage of the fact that nested words over a 2-stack call-return alphabet can be written as a string with one type of stack edges above the string and the other below the string, where the first type concerns the first stack and the other type concerns the second stack. In the 2-stack case, the edges do not intersect.

Note that a nested word needs not be well-matched. It might have pending calls, i.e., calls without matching return, as well as pending returns, i.e., returns that do not have a matching call. Therefore, the relations $\mu$ and its inverse $\mu^{-1}$ can be seen as partial maps $[n] \rightarrow[n]$, in the obvious manner. Moreover, observe that, given nested words 
$W=([n], \lessdot, \mu, \lambda)$ and $W^{\prime}=\left(\left[n^{\prime}\right], \lessdot^{\prime}, \mu^{\prime}, \lambda^{\prime}\right), n=n^{\prime} \wedge \lambda=\lambda^{\prime}$ implies $W=W^{\prime}$. It is therefore justified to represent $W$ as the string $\operatorname{string}(W):=\lambda(1) \ldots \lambda(n) \in \Sigma^{+}$. This naturally extends to sets $\mathcal{L}$ of nested words and we set $\operatorname{string}(\mathcal{L}):=\{\operatorname{string}(W) \mid W \in \mathcal{L}\}$. Vice versa, given a string $w \in \Sigma^{+}$, there is precisely one nested word $W$ over $\widetilde{\Sigma}$ such that $\operatorname{string}(W)=w$. This unique nested word is denoted nested $(w)$. For $L \subseteq \Sigma^{+}$, we let $\operatorname{nested}(L):=\{\operatorname{nested}(w) \mid w \in L\}$.

Example 2.4. Consider the 2-stack call-return alphabet $\widetilde{\Sigma}$ from Example 2.2, which was given by $\Sigma_{c}^{1}=\{a\}, \Sigma_{r}^{1}=\{\bar{a}\}, \Sigma_{c}^{2}=\{b\}, \Sigma_{r}^{2}=\{\bar{b}\}$, and $\Sigma_{\text {int }}=\emptyset$. Figure 2 depicts a nested word $W=([n], \lessdot, \mu, \lambda)$ over $\widetilde{\Sigma}$ with $n=10$. The straight arrows represent $\lessdot$, the curved arrows capture $\mu$ (those above the horizontal correspond to the first stack). For example, $(2,9) \in \mu$. Thus, $\mu(2)$ and $\mu^{-1}(9)$ are defined, whereas both $\mu^{-1}(7)$ and $\mu^{-1}(10)$ are not. In terms of visibly pushdown automata, this means that positions 7 and 10 are employed when the first/second stack is empty, respectively. Observe that $W=\operatorname{nested}(a b a b \bar{a} \bar{a} \bar{a} \bar{b} \bar{b} \bar{b})$ and $\operatorname{string}(W)=a b a b \bar{a} \bar{a} \bar{a} \bar{b} \bar{b} \bar{b}$.

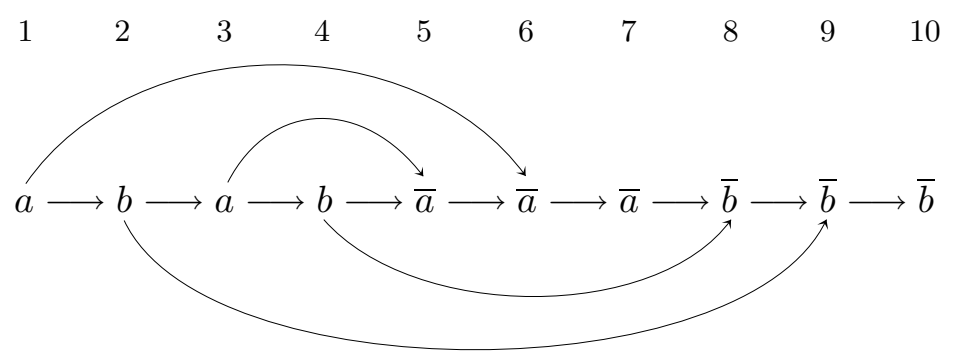

Figure 2: A nested word

We now turn to an automata model that is suited to nested words and, to some extent, is equivalent to MvPA. Our model is an extension of nested-word automata for one stack, which has been considered in [2], to multiple stacks. We also extend the model of [2] by calling states. If the state that is reached after executing some action $a$ is a calling state, then the corresponding run is accepting only if this $a$ is a call with a matching return (i.e., it is not pending). We will later see that this concept does not increase the expressive power of our automata but turns out to be a convenient tool when we translate logical formulas into automata.

Definition 2.5. A generalized multi-stack nested-word automaton (generalized MNWA) over $\widetilde{\Sigma}$ is a tuple $\mathcal{B}=\left(Q, \delta, Q_{I}, F, C\right)$ where

- $Q$ is the finite set of states,

- $Q_{I} \subseteq Q$ is the set of initial states,

- $F \subseteq Q$ is the set of final states,

- $C \subseteq Q$ is a set of calling states, and

- $\delta$ is a pair $\left\langle\delta_{1}, \delta_{2}\right\rangle$ of relations $\delta_{1} \subseteq Q \times \Sigma \times Q$ and $\delta_{2} \subseteq Q \times Q \times \Sigma_{r} \times Q$, which contain the transitions.

We call $\mathcal{B}$ a multi-stack nested-word automaton (MNWA) if $C=\emptyset$.

A (generalized) 2-stack nested-word automaton ((generalized) 2NWA) is a (generalized, respectively) MNWA that is defined over a 2-stack alphabet (i.e., $K=2$ ). 
B. BOLLIG

Intuitively, $\delta_{1}$ contains all the local and push transitions, as well as all the pop transitions that act on an empty stack (i.e., in terms of nested words and nested-word automata, those transitions that perform an action from $\Sigma_{r}$ that is not matched by a corresponding calling action). A run of $\mathcal{B}$ on a nested word $W=([n], \lessdot, \mu, \lambda)$ over $\widetilde{\Sigma}$ is a mapping $\rho:[n] \rightarrow Q$ such that $(q, \lambda(1), \rho(1)) \in \delta_{1}$ for some $q \in Q_{I}$, and, for all $i \in\{2, \ldots, n\}$, we have

$$
\left\{\begin{aligned}
\left(\rho\left(\mu^{-1}(i)\right), \rho(i-1), \lambda(i), \rho(i)\right) & \in \delta_{2} & & \text { if } \mu^{-1}(i) \text { is defined } \\
(\rho(i-1), \lambda(i), \rho(i)) & \in \delta_{1} & & \text { otherwise }
\end{aligned}\right.
$$

The run $\rho$ is accepting if $\rho(n) \in F$ and, for all $i \in[n]$ with $\rho(i) \in C, \mu(i)$ is defined. The language of $\mathcal{B}$, denoted by $\mathcal{L}(\mathcal{B})$, is the set of nested words from $\mathbb{N} \mathbb{W}(\widetilde{\Sigma})$ that allow for an accepting run of $\mathcal{B}$.

Recall that there is a one-to-one correspondence between strings and nested words. We let therefore $\mathcal{L}(\mathcal{A})$ with $\mathcal{A}$ an MvpA stand for the set nested $(L(\mathcal{A}))$.

Example 2.6. Consider again the 2-stack call-return alphabet $\widetilde{\Sigma}$ given by $\Sigma_{c}^{1}=\{a\}$, $\Sigma_{r}^{1}=\{\bar{a}\}, \Sigma_{c}^{2}=\{b\}, \Sigma_{r}^{2}=\{\bar{b}\}$, and $\Sigma_{i n t}=\emptyset$. In Example 2.2, we have seen that, for $L=\left\{(a b)^{n} \bar{a}^{n+1} \bar{b}^{n+1} \mid n \geq 1\right\}$, the iteration $L^{+}$is the language of some 2 vPA over $\widetilde{\Sigma}$. We can also specify a 2 NwA $\mathcal{B}=\left(\left\{q_{0}, \ldots, q_{4}\right\}, \delta,\left\{q_{0}\right\},\left\{q_{0}\right\}, \emptyset\right)$ over $\widetilde{\Sigma}$ such that $\mathcal{L}(\mathcal{B})=\operatorname{nested}\left(L^{+}\right)$. Note that $\mathcal{L}(\mathcal{B})$ will contain, for example, the nested word that is depicted in Figure 2, The transition relation $\delta$ is given as follows:

$$
\begin{aligned}
& \delta_{1}:\left(q_{0}, a, q_{2}\right) \quad \delta_{2}:\left(q_{2}, q_{3}, \bar{a}, q_{3}\right) \\
& \left(q_{2}, b, q_{1}\right) \quad\left(q_{3}, q_{4}, \bar{b}, q_{4}\right) \\
& \left(q_{1}, a, q_{2}\right) \quad\left(q_{1}, q_{4}, \bar{b}, q_{4}\right) \\
& \left(q_{2}, b, q_{3}\right) \\
& \left(q_{3}, \bar{a}, q_{4}\right) \\
& \left(q_{4}, \bar{b}, q_{0}\right)
\end{aligned}
$$

Similarly to Example 2.2, the finite-state control will ensure the general regular structure of a word without explicit "counting". This counting is then implicitly done by the relation $\delta_{2}$, which requires a matching call for a return. A graphical description of $\mathcal{B}$ is given in Figure 3. Hereby, a return transition with an adjoining set of states indicates that one state of this set must have been reached right after executing the corresponding call (in particular, the return must not be pending), whereas the remaining return transitions, $\left(q_{3}, \bar{a}, q_{4}\right)$ and $\left(q_{4}, \bar{b}, q_{0}\right)$, apply only to pending returns.

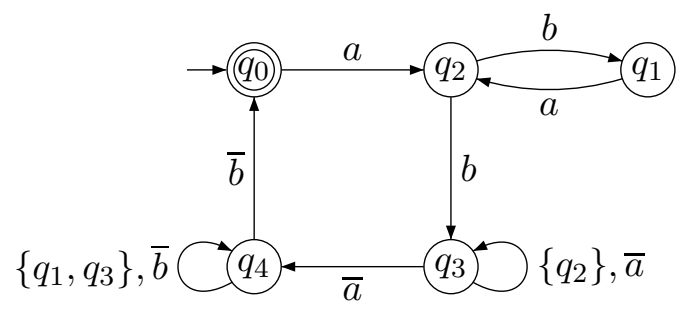

Figure 3: A 2NWA 
A general technique for a reduction from MvPA to MNWA and vice versa can be found below (Lemma 2.8).

We can show that the use of calling states does not increase the expressiveness of MnwA. Note that, however, the concept of calling states will turn out to be helpful when building the sphere automaton in Section 4.2 .

Lemma 2.7. For every generalized MNWA $\mathcal{B}$ over $\widetilde{\Sigma}$, there is an MNWA $\mathcal{B}^{\prime}$ over $\widetilde{\Sigma}$ such that $\mathcal{L}\left(\mathcal{B}^{\prime}\right)=\mathcal{L}(\mathcal{B})$.

Proof. In the construction of an MNWA, we exploit the following property of a nested word $W=([n], \lessdot, \mu, \lambda)$ : given $(i, j) \in \mu$, say, with $\lambda(i) \in \Sigma_{c}^{s}, \mu\left(i^{\prime}\right)$ is defined for all $i^{\prime} \in\{i+1, \ldots, j-1\}$ satisfying $\lambda\left(i^{\prime}\right) \in \Sigma_{c}^{s}$. Basically, $\mathcal{B}^{\prime}$ will simulate $\mathcal{B}$. In addition, whenever a calling state is assigned to a position labeled with an element from $\Sigma_{c}^{s}$, we will set a flag $\overline{\mathrm{b}}[s]=1$, which can only be resolved and turn into a final state $(\overline{\mathrm{b}}[s]=0)$ when a matching return position has been found. As any interim call position that concerns stack $s$ is matched anyway, the flags $\overline{\mathrm{b}}[s]$ in that interval are set to 2 . Thus, while a flag is 1 or 2 , there is still some unmatched calling position. Hence, a final state requires every flag to equal 0 , which also designates the initial state.

Let us become more precise and let $\mathcal{B}=\left(Q, \delta, Q_{I}, F, C\right)$ be a generalized MNwA. We determine the MNwa $\mathcal{B}^{\prime}=\left(Q^{\prime}, \delta^{\prime}, Q_{I}^{\prime}, F^{\prime}, \emptyset\right)$ by $Q^{\prime}=Q \times\{0,1,2\}^{[K]}, Q_{I}^{\prime}=Q_{I} \times\left\{(0)_{s \in[K]}\right\}$, $F^{\prime}=F \times\left\{(0)_{s \in[K]}\right\}$, and $\delta^{\prime}=\left\langle\delta_{1}^{\prime}, \delta_{2}^{\prime}\right\rangle$ where

- $\delta_{1}^{\prime}$ is the set of triples $\left((q, \overline{\mathrm{b}}), a,\left(q^{\prime}, \overline{\mathrm{b}}^{\prime}\right)\right) \in Q^{\prime} \times \Sigma \times Q^{\prime}$ such that $\left(q, a, q^{\prime}\right) \in \delta_{1}, q^{\prime} \in C$ implies $a \in \Sigma_{c}$, and, for every $s \in[K]$,

$$
\overline{\mathrm{b}}^{\prime}[s]= \begin{cases}2 & \text { if } \overline{\mathrm{b}}[s] \in\{1,2\} \\ 1 & \text { if } \overline{\mathrm{b}}[s]=0 \text { and } a \in \Sigma_{c}^{s} \text { and } q^{\prime} \in C \\ 0 & \text { otherwise }\end{cases}
$$

- $\delta_{2}^{\prime}$ is the set of quadruples $\left((p, \overline{\mathrm{c}}),(q, \overline{\mathrm{b}}), a,\left(q^{\prime}, \overline{\mathrm{b}}^{\prime}\right)\right) \in Q^{\prime} \times Q^{\prime} \times \Sigma_{r} \times Q^{\prime}$ such that $\left(p, q, a, q^{\prime}\right) \in$ $\delta_{2}, q^{\prime} \notin C$, and, for every $s \in[K]$,

$$
\overline{\mathrm{b}}^{\prime}[s]=\left\{\begin{array}{cc}
0 & \text { if } \overline{\mathrm{c}}[s]=1 \\
\overline{\mathrm{b}}[s] & \text { otherwise }
\end{array}\right.
$$

In fact, we can show that $\mathcal{L}(\mathcal{B})=\mathcal{L}\left(\mathcal{B}^{\prime}\right)$.

Note that the flag assignments depend deterministically on the input word and the states assigned to the positions. Let $W=([n], \lessdot, \mu, \lambda)$ be a nested word over $\widetilde{\Sigma}$.

Suppose $\rho$ to be an accepting run of $\mathcal{B}$ on $W$ and let $\widehat{\rho}:[n] \rightarrow\{0,1,2\}^{[K]}$ be the unique supplement of $\rho$ according to the flag construction. To verify that $(\rho, \widehat{\rho})$ is indeed an accepting run of $\mathcal{B}^{\prime}$ on $W$, we need to show that $\widehat{\rho}(n)[s]=0$ for all $s \in[K]$. So let $s \in[K]$. If there is no $i \in[n]$ such that $\lambda(i) \in \Sigma_{c}^{s}$ and $\rho(i) \in C$, then we clearly have $\widehat{\rho}(n)[s]=0$, as the flag for stack $s$ never changes its value during the run. If the flag changes its value from 0 to 1 , then this happens at a position $i \in[n]$ such that $\lambda(i) \in \Sigma_{c}^{s}$ and $\rho(i) \in C$. As $\rho$ is an accepting run of $\mathcal{B}$ on $W$, there is $j \in[n]$ such that $(i, j) \in \mu$. By construction of $\mathcal{B}^{\prime}$, $\widehat{\rho}(i)[s]=1, \widehat{\rho}\left(i^{\prime}\right)[s]=2$ for all $i^{\prime} \in\{i+1, \ldots, j-1\}$, and $\widehat{\rho}(j)[s]=0$. Thus, we finally have $\widehat{\rho}(n)[s]=0$.

Conversely, let $\rho:[n] \rightarrow Q$ and $\widehat{\rho}:[n] \rightarrow\{0,1,2\}^{[K]}$ be mappings such that $(\rho, \widehat{\rho})$ is an accepting run of $\mathcal{B}^{\prime}$ on $W$. Clearly, $\rho$ is a run of $\mathcal{B}$ on $W$. So let us verify that it is accepting. 
First, observe that $\rho(n) \in F$. So suppose $i \in[n]$ such that $\rho(i)$ is a calling state. According to the construction of $\mathcal{B}^{\prime}, \lambda(i) \in \Sigma_{c}^{s}$ for some $s$. Moreover, we have $\widehat{\rho}(i)[s]=\{1,2\}$. As $\widehat{\rho}(n)[s]=0$, there must be $i^{\prime} \leq i$ and $j^{\prime}>i$ such that $\lambda\left(i^{\prime}\right) \in \Sigma_{c}^{s}$ and $\left(i^{\prime}, j^{\prime}\right) \in \mu$. This implies that $\mu(i)$ is indeed defined so that we can conclude that $\rho$ is an accepting run of $\mathcal{B}$ on $W$.

The flag construction from the previous proof is illustrated in Figure 4, where we assume a run on the nested word such that every state associated with a symbol from $\{a, b\}$ is a calling state.

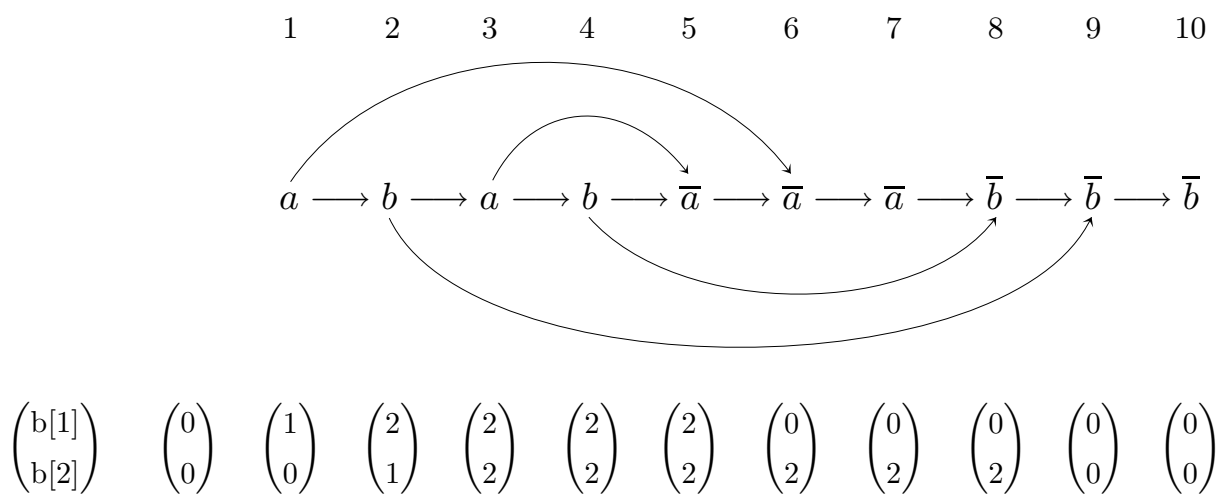

Figure 4: The flag construction

Lemma 2.8. Let $\mathcal{L} \subseteq \mathbb{N} \mathbb{W}(\widetilde{\Sigma})$ be a set of nested words over $\widetilde{\Sigma}$. The following are equivalent:

(1) There is an MvPA $\mathcal{A}$ over $\widetilde{\Sigma}$ such that $\mathcal{L}(\mathcal{A})=\mathcal{L}$.

(2) There is an MNWA $\mathcal{B}$ over $\widetilde{\Sigma}$ such that $\mathcal{L}(\mathcal{B})=\mathcal{L}$.

Proof. Given an Mvpa $\mathcal{A}=\left(Q, \Gamma, \delta, Q_{I}, F\right)$, we define an Mnwa $\mathcal{B}=\left(Q^{\prime}, \delta^{\prime}, Q_{I}^{\prime}, F^{\prime}, \emptyset\right)$ with $\mathcal{L}(\mathcal{A})=\mathcal{L}(\mathcal{B})$ as follows: $Q^{\prime}=Q \times \Gamma, Q_{I}^{\prime}=Q_{I} \times\{\perp\}, F^{\prime}=F \times \Gamma$, and $\delta^{\prime}=\left\langle\delta_{1}^{\prime}, \delta_{2}^{\prime}\right\rangle$ where

- $\delta_{1}^{\prime}$ is the set of triples $\left((q, A), a,\left(q^{\prime}, A^{\prime}\right)\right) \in Q^{\prime} \times \Sigma \times Q^{\prime}$ such that $\left(q, a, A^{\prime}, q^{\prime}\right) \in \delta_{c}$, $\left(q, a, q^{\prime}\right) \in \delta_{\text {int }}$, or $\left(q, a, \perp, q^{\prime}\right) \in \delta_{r}$, and

- $\delta_{2}^{\prime}$ is the set of quadruples $\left((p, B),(q, A), a,\left(q^{\prime}, A^{\prime}\right)\right) \in Q^{\prime} \times Q^{\prime} \times \Sigma \times Q^{\prime}$ such that $\left(q, a, B, q^{\prime}\right) \in \delta_{r}$.

The idea is that the stack symbol associated with a transition is incorporated into the state of the MNWA. When an internal or unmatched return action is performed, then we may chose an arbitrary stack symbol, as it will not be reconsidered later in the run.

For the converse direction, let $\mathcal{B}=\left(Q, \delta, Q_{I}, F, \emptyset\right)$ be an Mnwa. Consider the MvpA $\mathcal{A}=\left(Q, Q \bullet\{\perp\}, \delta^{\prime}, Q_{I}, F\right)$ where $\delta^{\prime}=\left\langle\delta_{c}^{\prime}, \delta_{r}^{\prime}, \delta_{\text {int }}^{\prime}\right\rangle$ is given by

- $\delta_{c}^{\prime}=\left\{\left(q, a, q^{\prime}, q^{\prime}\right) \mid\left(q, a, q^{\prime}\right) \in \delta_{1} \cap\left(Q \times \Sigma_{c} \times Q\right)\right\}$,

- $\delta_{i n t}^{\prime}=\delta_{1} \cap\left(Q \times \Sigma_{i n t} \times Q\right)$, and

- $\delta_{r}^{\prime}$ is the set of tuples $\left(q, a, A, q^{\prime}\right) \in Q \times \Sigma_{r} \times \Gamma \times Q$ such that either $\left(q, a, q^{\prime}\right) \in \delta_{1}$ and $A=\perp$, or $\left(A, q, a, q^{\prime}\right) \in \delta_{2}$.

Here, we need to ensure that, when $\mathcal{A}$ performs a matched return action, we can access the state that $\mathcal{B}$ has associated with the corresponding call. To this aim, $\mathcal{A}$ just pushes the state onto the stack so that it becomes accessible when the corresponding return is executed. It is straightforward to show that $\mathcal{L}(\mathcal{A})=\mathcal{L}(\mathcal{B})$. 


\section{Monadic Second-Order Logic and Hanf's Theorem}

3.1. Monadic Second-Order Logic over Relational Structures. We fix supplies of first-order variables $x, y, \ldots$ and second-order variables $X, Y, \ldots$ Let $\tau$ be a function-free signature. The set $\mathrm{MSO}(\tau)$ of monadic second-order (MSO) formulas over $\tau$ is given by the following grammar:

$$
\varphi::=P\left(x_{1}, \ldots, x_{m}\right)\left|x_{1}=x_{2}\right| x \in X|\neg \varphi| \varphi_{1} \vee \varphi_{2}|\exists x \varphi| \exists X \varphi
$$

Hereby, $m \geq 1, P \in \tau$ is an $m$-ary predicate symbol, the $x_{k}$ and $x$ are first-order variables, and $X$ is a second-order variable. Moreover, we will make use of the usual abbreviations such as $\varphi_{1} \wedge \varphi_{2}$ for $\neg\left(\neg \varphi_{1} \vee \neg \varphi_{2}\right), \varphi_{1} \rightarrow \varphi_{2}$ for $\neg \varphi_{1} \vee \varphi_{2}$, etc. Given a $\tau$-structure $\mathfrak{A}$ with universe $A$, a formula $\varphi\left(x_{1}, \ldots, x_{m}, X_{1}, \ldots, X_{n}\right) \in \operatorname{MSO}(\tau)$ with free variables in $\left\{x_{1}, \ldots, x_{m}, X_{1}, \ldots, X_{n}\right\},\left(u_{1}, \ldots, u_{m}\right) \in A^{m}$, and $\left(U_{1}, \ldots, U_{n}\right) \in\left(2^{A}\right)^{n}$, we write, as usual, $\mathfrak{A} \models \varphi\left[u_{1}, \ldots, u_{m}, U_{1}, \ldots, U_{n}\right]$ if $\mathfrak{A}$ satisfies $\varphi$ when assigning $\left(u_{1}, \ldots, u_{m}\right)$ to $\left(x_{1}, \ldots, x_{m}\right)$ and $\left(U_{1}, \ldots, U_{n}\right)$ to $\left(X_{1}, \ldots, X_{n}\right)$.

Let us identify some important fragments of $\operatorname{MSO}(\tau)$. The set $\mathrm{FO}(\tau)$ of first order (FO) formulas over $\tau$ comprises those formulas from $\operatorname{MSO}(\tau)$ that do not contain any second-order quantifier. Furthermore, an existential MSO (EMSO) formula is of the form $\exists X_{1} \ldots \exists X_{n} \varphi$ with $\varphi \in \operatorname{FO}(\tau)$. The corresponding class of formulas is denoted $\operatorname{EMSO}(\tau)$. More generally, given $m \geq 1$, we denote by $\boldsymbol{\Sigma}_{m}(\tau)$ the set of formulas of the form $\exists \overline{X_{1}} \forall \overline{X_{2}} \ldots \exists / \forall \overline{X_{m}} \varphi$ where $\varphi \in \mathrm{FO}(\tau)$ and the $\overline{X_{k}}$ are blocks of second-order variables, possibly empty or of different length.

We will later make use of the notion of definability relative to a class of structures. Let $\mathcal{F} \subseteq \operatorname{MSO}(\tau)$ be a class of formulas and $\mathcal{L}, \mathcal{C}$ be sets of $\tau$-structures. We say that $\mathcal{L}$ is $\mathcal{F}$-definable relative to $\mathcal{C}$ if there is a sentence (i.e., a formula without any free variables) $\varphi \in \mathcal{F}$ such that $\mathcal{L}$ is the set of $\tau$-structures $\mathfrak{A} \in \mathcal{C}$ such that $\mathfrak{A} \models \varphi$.

3.2. Hanf's Theorem for Nested Words, and Spheres. We will now provide a signature that allows us to specify MSO properties of nested words. Let $\widetilde{\Sigma}$ be a call-return alphabet. We define $\tau_{\widetilde{\Sigma}}$ to be the signature $\left\{\lambda_{a} \mid a \in \Sigma\right\} \cup\{\lessdot, \mu\}$ with $\lambda_{a}$ a unary and $\lessdot$ and $\mu$ binary predicate symbols. We write the MSO formula $\lambda_{a}(x)$ as $\lambda(x)=a$ and the formula $\lessdot\left(x_{1}, x_{2}\right)$ as $x_{1} \lessdot x_{2}$. MSO formulas over $\tau_{\widetilde{\Sigma}}$ can be canonically interpreted over nested words $([n], \lessdot, \mu, \lambda) \in \mathbb{N} \mathbb{W}(\widetilde{\Sigma})$, as $\lambda$ can be seen as a collection of unary relations $\lambda_{a}=\{i \in[n] \mid \lambda(i)=a\}$ where $a \in \Sigma$. Thus, nested words over $\widetilde{\Sigma}$ are actually $\tau_{\widetilde{\Sigma}}$-structures. A sample MSO formula over $\tau_{\widetilde{\Sigma}}$ such that $\Sigma=\{a, b\}$ is $\forall x \forall y(\lambda(x)=a \wedge \mu(x, y) \rightarrow \lambda(y)=b)$. It expresses that every matching pair with a calling $a$ has a $b$-labeled return position. Given a sentence $\varphi \in \operatorname{MSO}\left(\tau_{\widetilde{\Sigma}}\right)$, we denote by $\mathcal{L}(\varphi)$ the set of nested words over $\widetilde{\Sigma}$ that satisfy $\varphi$, i.e., $\mathcal{L}(\varphi)=\{W \in \mathbb{N} \mathbb{W}(\widetilde{\Sigma}) \mid W \models \varphi\}$.

Over nested words (more generally, structures of bounded degree), FO formulas enjoy a normal form in terms of local formulas. A formula $\varphi(x) \in \mathrm{FO}\left(\tau_{\widetilde{\Sigma}}\right)$ with one free variable $x$ is said to be local if there is $r \in \mathbb{N}$ such that, in every subformula $\exists y \psi$ of $\varphi, \psi$ is of the form $(d(x, y) \leq r) \wedge \chi$. Hereby, the formula $d(x, y) \leq r$ has the expected meaning and can be obtained inductively. Informally, the truth of a local formula $\varphi(x)$ depends only on the local neighborhood around $x$.

Next, we state Hanf's locality theorem in terms of nested words. It actually applies to general classes of structures of bounded degree. 
Theorem 3.1 (Hanf [11]). Let $\varphi \in \mathrm{FO}\left(\tau_{\widetilde{\Sigma}}\right)$ be a sentence. There is a positive Boolean combination $\psi$ of formulas of the form

$$
\exists^{=t} x \chi(x) \text { and } \exists^{>t} x \chi(x)
$$

where $t \in \mathbb{N}$ and $\chi \in \mathrm{FO}\left(\tau_{\widetilde{\Sigma}}\right)$ is local (with the obvious meaning of the quantifiers $\exists=t$ and $\exists^{>t}$; note that there might occur different thresholds $t$ in $\psi$ ) such that, for every nested word $W \in \mathbb{N} \mathbb{W}(\widetilde{\Sigma})$, we have

$$
W \models \varphi \text { iff } W \models \psi
$$

Moreover, $\psi$ can be computed effectively and in elementary time.

For a comprehensive proof of this theorem, see, for example, $[16,20]$. However, these proofs are not effective, whereas the original proof by Hanf is effective. It is crucial to note that Hanf's Theorem applies to the case of nested words as we deal with a class of structures of bounded degree (see below for a formal definition). Indeed, there is a uniform bound on the degree of nested words.

Let $\mathfrak{A}=(N, \lessdot, \mu, \lambda, \ldots)$ and $\mathfrak{A}^{\prime}=\left(N^{\prime}, \lessdot^{\prime}, \mu^{\prime}, \lambda^{\prime}, \ldots\right)$ be tuples such that $(N, \lessdot, \mu, \lambda)$ and $\left(N^{\prime}, \lessdot^{\prime}, \mu^{\prime}, \lambda^{\prime}\right)$ are $\tau_{\widetilde{\Sigma}}$-structures. For $i, j \in N$ and $i^{\prime}, j^{\prime} \in N^{\prime}$, we write $(i, j) \sqsubseteq \check{\mathscr{A}}^{\prime}\left(i^{\prime}, j^{\prime}\right)$ if $\lambda(i)=\lambda^{\prime}\left(i^{\prime}\right), \lambda(j)=\lambda^{\prime}\left(j^{\prime}\right),(i, j) \in \lessdot \operatorname{implies}\left(i^{\prime}, j^{\prime}\right) \in \lessdot^{\prime}$, and $(i, j) \in \mu$ implies $\left(i^{\prime}, j^{\prime}\right) \in \mu^{\prime}$. Theorem 3.1 suggests that, over nested words, the validity of an FO formula in a nested word depends on the local neighborhoods of the latter. This leads to the notion of a sphere, which will actually play a central role in the remainder of this paper. A sphere of radius $r \in \mathbb{N}$ includes elements whose distance from a distinguished sphere center is bounded by $r$. Given $i, j \in N$, the distance $d_{\mathfrak{A}}(i, j)$ of $i$ and $j$ in $\mathfrak{A}$ is the minimal length of a path from $i$ to $j$ in the Gaifman graph of $(N, \lessdot, \mu, \lambda)$. The Gaifman graph of $(N, \lessdot, \mu, \lambda)$ is defined to be the undirected graph $(N$, Arcs $)$ where $(i, j) \in \operatorname{Arcs}$ iff $(i, j) \in \lessdot \cup \mu \cup \lessdot^{-1} \cup \mu^{-1}[16]$. In particular, we have $d_{\mathfrak{A}}(i, i)=0$. If $d_{\mathfrak{A}}(i, j)=1$, we also write $i \leftrightarrow \mathfrak{A} j$. We write $i \rightarrow_{\mathfrak{A}} j$ if $(i, j) \in \lessdot \cup \mu$. The degree of a $\tau_{\widetilde{\Sigma}}$-structure is said to be bounded by some natural number $B$ if the degree of its Gaifman graph is bounded by $B$. Observe that the degree of a nested word is bounded by 3 , which is therefore a uniform bound for the class $\mathbb{N W V}(\widetilde{\Sigma})$.

Let $\mathfrak{B}=(N, \lessdot, \mu, \lambda)$ be a $\tau_{\widetilde{\Sigma}}$-structure, $r \in \mathbb{N}$, and $i \in N$. The $r$-sphere of $\mathfrak{B}$ around $i$, which we denote by $r$-Sph $(\mathfrak{B}, i)$, is basically the substructure of $\mathfrak{B}$ induced by the new universe $\left\{j \in N \mid d_{\mathfrak{B}}(i, j) \leq r\right\}$, but extended by the constant $i$ as a distinguished element, called the sphere center. Given an isomorphism type $S$ of an $r$-sphere, we let $|\mathfrak{B}|_{S}:=\mid\{i \in$ $N \mid S \cong r-\operatorname{Sph}(\mathfrak{B}, i)\} \mid$ denote the number of points in $\mathfrak{B}$ that realize $S$. For an example, consider Figure 5, showing a nested word $W$ and the 2-sphere of $W$ around $i=10$ where the sphere center is marked as a rectangle. Note that 2 - $\operatorname{Sph}(W, 10) \cong 2-\operatorname{Sph}(W, 14)$ and $|W|_{2-\operatorname{Sph}(W, 10)}=2$.

We denote by Spheres $_{r}(\widetilde{\Sigma})$ the set of (isomorphism types of) $r$-spheres that arise from nested words over $\widetilde{\Sigma}$, i.e.,

$$
\operatorname{Spheres}_{r}(\widetilde{\Sigma}):=\{r-\operatorname{Sph}(W, i) \mid W \in \mathbb{N} \mathbb{W}(\widetilde{\Sigma}) \text { and } i \text { is a node of } W\} .
$$

Note that $\operatorname{Spheres}_{r}(\widetilde{\Sigma})$ is finite up to isomorphism, which is crucial for the constructions in Section 4 . 

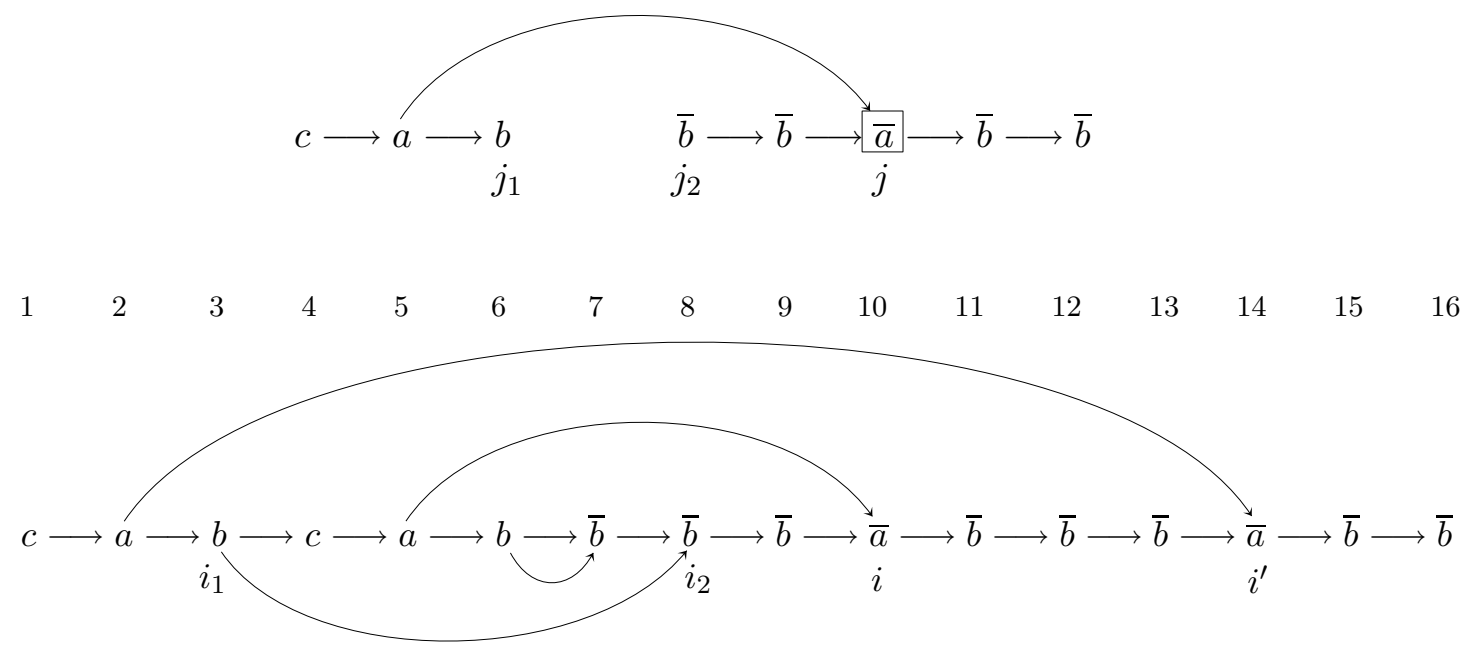

Figure 5: A 2-shpere embedded into a nested word

\section{2-Stack Visibly Pushdown Automata vs. Logic}

In this section, we focus on 2VPA. So let us fix a 2-stack call-return alphabet $\widetilde{\Sigma}=$ $\left\langle\left\{\left(\Sigma_{c}^{1}, \Sigma_{r}^{1}\right),\left(\Sigma_{c}^{2}, \Sigma_{r}^{2}\right)\right\}, \Sigma_{\text {int }}\right\rangle$.

4.1. The Main Result. The key connection between FO logic and 2vPA/2NWA is provided by the following proposition, which states the existence of an automaton that computes the sphere around any node of a nested word.

Proposition 4.1. Let $r$ be any natural number. There are a generalized 2NWA $\mathcal{B}_{r}=$ $\left(Q, \delta, Q_{I}, F, C\right)$ over $\widetilde{\Sigma}$ and a mapping $\eta: Q \rightarrow$ Spheres $_{r}(\widetilde{\Sigma})$ such that

- $\mathcal{L}\left(\mathcal{B}_{r}\right)=\mathbb{N} \mathbb{V}(\widetilde{\Sigma})$ (i.e., every nested word admits an accepting run of $\mathcal{B}_{r}$ ), and

- for every nested word $W \in \mathbb{N} \mathbb{W}(\widetilde{\Sigma})$, every accepting run $\rho$ of $\mathcal{B}_{r}$ on $W$, and every node $i$ of $W$, we have $\eta(\rho(i)) \cong r-\operatorname{Sph}(W, i)$.

Before we turn towards the proof of this statement, we will first show how Proposition 4.1 can be used to establish expressive equivalence of 2VPA and EMSO logic.

Lemma 4.2. Let $r, t \in \mathbb{N}$ and let $S \in$ Spheres $_{r}(\widetilde{\Sigma})$ be an $r$-sphere in some nested word over $\widetilde{\Sigma}$. There are generalized $2 \mathrm{NWA} \mathcal{B}^{1}$ and $\mathcal{B}^{2}$ over $\widetilde{\Sigma}$ such that $\mathcal{L}\left(\mathcal{B}^{1}\right)=\{W \in \mathbb{N} \mathbb{W}(\widetilde{\Sigma}) \mid$ $\left.|W|_{S}=t\right\}$ and $\mathcal{L}\left(\mathcal{B}^{2}\right)=\left\{\left.W \in \mathbb{N W}(\widetilde{\Sigma})|| W\right|_{S}>t\right\}$.

Proof. In both cases, we start from the generalized 2NwA $\mathcal{B}_{r}=\left(Q, \delta, Q_{I}, F, C\right)$ and the mapping $\eta: Q \rightarrow$ Spheres $_{r}(\widetilde{\Sigma})$ from Proposition 4.1. For $k=1,2$, we obtain $\mathcal{B}^{k}$ by extending the state space with a counter that, using $\eta$, counts the number of realizations of $S$ up to $t+1$. The new set of initial states is thus in both cases $Q_{I} \times\{0\}$. However, the set of final states of $\mathcal{B}^{1}$ is $F \times\{t\}$, the one of $\mathcal{B}^{2}$ is $F \times\{t+1\}$. 
We are now prepared to state the first main result of this paper.

Theorem 4.3. Let $\mathcal{L} \subseteq \mathbb{N} \mathbb{W}(\widetilde{\Sigma})$ be a set of nested words over the 2-stack call-return alphabet $\widetilde{\Sigma}$. Then, the following are equivalent:

(1) There is a 2VPA $\mathcal{A}$ over $\widetilde{\Sigma}$ such that $\mathcal{L}(\mathcal{A})=\mathcal{L}$.

(2) There is a sentence $\varphi \in \operatorname{EMSO}\left(\tau_{\widetilde{\Sigma}}\right)$ such that $\mathcal{L}(\varphi)=\mathcal{L}$.

Both directions are effective. In particular, the 2VPA that we construct for a given EMSO sentence can be computed in elementary time, and its size is elementary in the size of the formula.

Proof. To prove $(1) \rightarrow(2)$, one can perform a standard construction of an EMSO formula from a $2 \mathrm{NWA}$, where the latter can be extracted from the given $2 \mathrm{VPA}$ according to Lemma 2.8. Basically, the formula "guesses" a possible run on the input word in terms of existentially quantified second-order variables and then verifies, in its first-order fragment, that we actually deal with a run that is accepting.

So let us directly prove $(2) \rightarrow(1)$ and let $\varphi=\exists X_{1} \ldots \exists X_{m} \psi\left(X_{1}, \ldots, X_{m}\right) \in \operatorname{EMSO}\left(\tau_{\widetilde{\Sigma}}\right)$ be a sentence with $\psi\left(X_{1}, \ldots, X_{m}\right) \in \mathrm{FO}\left(\tau_{\widetilde{\Sigma}}\right)$ (we suppose $m \geq 1$ ). We define a new 2 -stack call-return alphabet

$$
\widehat{\Sigma}=\left\langle\left\{\left(\Sigma_{c}^{1} \times 2^{[m]}, \Sigma_{r}^{1} \times 2^{[m]}\right),\left(\Sigma_{c}^{2} \times 2^{[m]}, \Sigma_{r}^{2} \times 2^{[m]}\right)\right\}, \Sigma_{\text {int }} \times 2^{[m]}\right\rangle
$$

where $2^{[m]}$ shall denote the powerset of $[m]$. From $\psi$, we obtain an FO formula $\psi^{\prime}$ over $\tau_{\widehat{\Sigma}}$ by replacing each occurrence of $\lambda(x)=a$ with $\bigvee_{M \in 2^{[m]}} \lambda(x)=(a, M)$ and each occurrence of $x \in X_{k}$ with $\bigvee_{a \in \Sigma, M \in 2^{[m]}} \lambda(x)=(a, M \cup\{k\})$. We set $\mathcal{L} \subseteq \mathbb{N} \mathbb{W}(\widehat{\Sigma})$ to be the set of nested words that satisfy $\psi^{\prime}$. From Hanf's Theorem (Theorem 3.1), we know that $\mathcal{L}$ is the language of a positive Boolean combination of formulas of the form $\exists^{=t} x \chi(x)$ and $\exists^{>t} x \chi(x)$ where $\chi$ is local. It is easy to see that the class of nested-word languages that are recognized by generalized 2NWA is closed under union and intersection. Thus, the validity of one such basic formula can be checked by a generalized 2NWA due to Lemma 4.2 . We deduce that there is a generalized 2NWA $\mathcal{B}^{\prime}$ over $\widetilde{\Sigma}$ recognizing $\mathcal{L}$.

Now, to check whether some nested word from $\mathbb{N} \mathbb{W}(\widetilde{\Sigma})$ satisfies $\varphi$, a generalized 2NWA $\mathcal{B}$ with $\mathcal{L}(\mathcal{B})=\mathcal{L}(\varphi)$ will guess an additional labeling for each node in terms of an element from $2^{[m]}$ and then simulate $\mathcal{B}^{\prime}$. By Lemma 2.7 and Lemma 2.8, we finally obtain a 2VPA $\mathcal{A}$ such that $\mathcal{L}(\mathcal{A})=\mathcal{L}(\varphi)$.

4.2. Proof of Proposition 4.1. We now turn to the proof of Proposition 4.1. In each state, the generalized 2NWA $\mathcal{B}_{r}$ will guess the current sphere as well as spheres of nodes nearby and the current position in these additional spheres. Adding some global information allows us to locally check whether all the guesses are correct. The rest of this section is devoted to the construction of $\mathcal{B}_{r}$ and a corresponding mapping $\eta$ to prove Proposition 4.1.

4.2.1. The Construction. Recall that $\operatorname{Spheres}_{r}(\widetilde{\Sigma})$ denotes the set of all the $r$-spheres that arise from nested words, i.e., Spheres $r(\widetilde{\Sigma})=\{r$-Sph $(W, i) \mid W$ is a nested word and $i$ is a position in $W\}$. An extended $r$-sphere over $\widetilde{\Sigma}$ is a tuple $E=(N, \lessdot, \mu, \lambda, \gamma, \alpha, c o l)$ where $\operatorname{core}(E):=(N, \lessdot, \mu, \lambda, \gamma) \in$ Spheres $_{r}(\widetilde{\Sigma})$ (in particular, $\left.\gamma \in N\right), \alpha \in N$, and col $\in[\# C o l]$ with $\#$ Col $=4 \cdot \operatorname{maxSize}(r)^{2}+1$ where $\operatorname{maxSize}(r)$ is the maximal size of an $r$-sphere, i.e., $\operatorname{maxSize}(r)=\max \left\{|N| \mid(N, \lessdot, \mu, \lambda, i) \in \operatorname{Spheres}_{r}(\widetilde{\Sigma})\right\}$. We say that $\alpha$ is the active 
node of $E$ and col is its color. Strictly speaking, $(N, \lessdot, \mu, \lambda, \gamma, \alpha, c o l)$ is not a mathematical structure, as col does not refer to an element of $N$. We introduced the function core to extract a mathematical structure from an extended sphere, which will allow us to deal with notions such as isomorphism.

Let eSpheres $_{r}(\widetilde{\Sigma})$ denote the set of all the (isomorphism classes of) extended spheres over $\widetilde{\Sigma}$. For an extended sphere $E=(N, \lessdot, \mu, \lambda, \gamma, \alpha, \mathrm{col})$ and an element $i \in N$, we denote by $E[i]$ the extended sphere $(N, \lessdot, \mu, \lambda, \gamma, i, c o l)$, i.e., the extended sphere that we obtain by replacing the active node $\alpha$ with $i$.

The idea of the construction of the generalized 2NWA $\mathcal{B}_{r}$ is the following: A state $\mathcal{E}$ of $\mathcal{B}_{r}$ is a set of extended spheres, which reflect the "environment" of a node that $\mathcal{E}$ is assigned to. Now suppose that, in a run of $\mathcal{B}_{r}$ on a nested word $\widetilde{W}=([\widetilde{n}], \widetilde{\leftarrow}, \widetilde{\mu}, \widetilde{\lambda}), \mathcal{E}$ is assigned to a position $i \in[\widetilde{n}]$ and contains $E=(N, \lessdot, \mu, \lambda, \gamma, \alpha, c o l)$. If the run is accepting, this will mean that the environment of $i$ in $\widetilde{W}$ looks like the environment of $\alpha$ in $E$. In particular, $\mathcal{E}$ will contain exactly one extended sphere $E=(N, \lessdot, \mu, \lambda, \gamma, \alpha$, col $)$ such that $\gamma$ and $\alpha$ coincide, meaning that $r-\operatorname{Sph}(\widetilde{W}, i) \cong(N, \lessdot, \mu, \lambda, \gamma)$. This is illustrated in Figure 6 depicting a nested word and a step of a run of the sphere automaton for $r=1$ on this word. States $\mathcal{E}$ and $\mathcal{E}^{\prime}$ are assigned to positions 4 and 5, respectively. Each state is a set of extended spheres. For clarity, however, we will neglect colors in the example. The sphere center is, as usual, depicted as a rectangle; the active node is marked as a circle. Observe that each state contains precisely one extended sphere in which the sphere center and the active node are identical. These are $E_{1} \in \mathcal{E}$, and, respectively, $E_{2}^{\prime} \in \mathcal{E}^{\prime}$. Indeed, $E_{1}$ corresponds to the 1 -sphere of the nested word around 4 , while $E_{2}^{\prime}$ reflects the 1 -sphere around 5 .

Of course, $\mathcal{B}_{r}$ has to locally guess the environment of a position. But how can we ensure that a guess is correct? Obviously, we have to pass a local guess to each neighboring position in $\widetilde{W}$. So suppose again that a state $\mathcal{E}$ containing $E=(N, \lessdot, \mu, \lambda, \gamma, \alpha, \operatorname{col})$ is assigned to a node $i$ of $\widetilde{W}$. As $\alpha$ shall correspond to $i$, we need to ensure that $\lambda(\alpha)=\widetilde{\lambda}(i)$ (this will be taken care of by item (2) in the definition of the transition relation below). Now suppose that $\alpha$ has a $\lessdot$-successor $j \in N$, i.e., $\alpha \lessdot j$. Then, we have to guarantee that $i<\widetilde{n}$. This is done by simply excluding $\mathcal{E}$ from the set of final states (in Figure 6 , neither $\mathcal{E}$ nor $\mathcal{E}^{\prime}$ are final states). Moreover, $j$ should correspond to $i+1$, which is ensured by passing $E[j]$ to the state that will be assigned to $i+1$ (see item (7); in Figure 6, $\mathcal{E}^{\prime}$ must therefore contain $E_{1}[j]$ where $j$ is the $\lessdot$-successor of the active node of $E_{1}$, and we actually have $\left.E_{1}^{\prime} \cong E_{1}[j]\right)$. On the other hand, if $i$ has a $\lessdot$-successor, then $\alpha$ must have a $\lessdot$-successor $j$ as well such that $E[j]$ belongs to the state that will be assigned to $i+1$. Observe that this rule applies unless $d_{E}(\gamma, \alpha)=r$, as then $i+1$ lies out of the area of responsibility of $E$ (see item (5)). Similar requirements have to be considered wrt. potential $\lessdot-/ \lessdot$-predecessors (see (3), (4), and (6)), as well as wrt. the relations $\mu$ and $\widetilde{\mu}$ (see $\left(3^{\prime}\right)-\left(7^{\prime}\right)$ ). One difficulty in our construction, however, is to guarantee the lack of an edge. So assume the extended sphere $E$ is the one given by Figure 5 with $j_{1}$ as the active node. Let us neglect colors for the moment. Suppose furthermore that $\widetilde{W}$ is the nested word from Figure 5, below the sphere. Then, an accepting run $\rho$ of $\mathcal{B}_{r}$ on $\widetilde{W}$ will assign to $i_{1}$ a state that contains $E$ (modulo some coloring). Moreover, the state assigned to $i$ will contain $E[j]$, where the sphere center and the active node coincide. We observe that, in $E$, the node $j_{1}$ is maximal. In particular, there is no $\mu$-edge between $j_{1}$ and $j_{2}$. This should be reflected in $\widetilde{W}$. A first idea to guarantee this might be to just prevent $\rho\left(i_{2}\right)$ from containing the extended sphere 

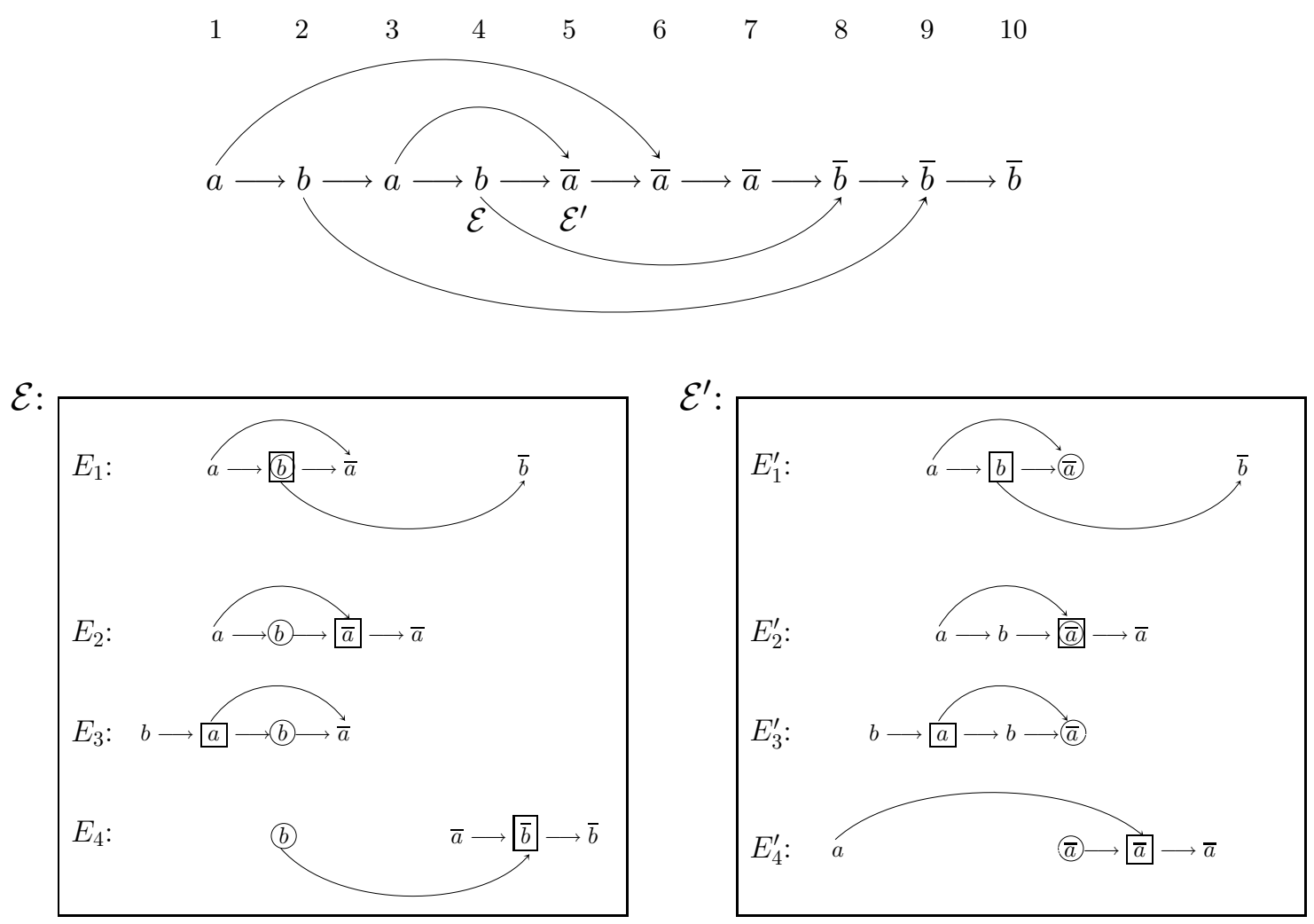

Figure 6: A step of the sphere automaton

$E\left[j_{2}\right]$ (note that $\left.\left(i_{1}, i_{2}\right) \in \widetilde{\mu}\right)$. This is, however, too restrictive. Actually, $\left(r-\operatorname{Sph}(\widetilde{W}, i), i_{2}\right)$ and $E\left[j_{2}\right]$ are isomorphic (neglecting the coloring of $E$ ) so that $\rho\left(i_{2}\right)$ must contain $E\left[j_{2}\right]$. The solution is already present in terms of the coloring of extended spheres. More precisely, $\rho\left(i_{2}\right)$ is allowed to carry $E\left[j_{2}\right]$ as soon as it has a color that is different from the color of the extended sphere $E\left[j_{1}\right]$ assigned to $i_{1}$. Roughly speaking, there might be isomorphic spheres in $\widetilde{W}$ that are overlapping. To consider them simultaneously, they are thus equipped with distinct colors.

The construction we obtain following the above ideas indeed allows us to infer, from an accepting run assigning a state $\mathcal{E}$ to a node $i$, the $r$-sphere around $i$. As mentioned above, we simply consider the (unique up to isomorphism) extended sphere $(N, \lessdot, \mu, \lambda, \gamma, \alpha, c o l)$ contained in $\mathcal{E}$ such that $\gamma=\alpha$. Then, $(N, \lessdot, \mu, \lambda, \gamma)$ is indeed the sphere of interest (recall that, in Figure 6 , these are $E_{1}$ for $\mathcal{E}$ and $E_{2}^{\prime}$ for $\mathcal{E}^{\prime}$ if we ignore active nodes and colors).

It is not obvious that the above ideas really do work, all the less as the construction will apply to nested words over two stacks, but no longer to nested words over more than two stacks. After all, the key argument will be provided by Proposition 4.5, stating an important property of nested words over two stacks. Intuitively, it states the following: Suppose that, in a nested word, there is an acyclic path from a node $i$ to another node $i^{\prime}$, and suppose this path is of a certain type $w$ (recording the labelings and edges seen in the path). Then, applying the same path several times will never lead back to $i$. This is finally the reason why a cycle in an extended sphere that occurs in a run on a nested word $\widetilde{W}$ is in fact simulated by $\widetilde{W}$. 
Let us formally construct the generalized 2NWA $\mathcal{B}_{r}=\left(Q, \delta, Q_{I}, F, C\right)$. An element of $Q$ is a subset $\mathcal{E}$ of $\operatorname{eSpheres}_{r}(\widetilde{\Sigma})$ such that either $\mathcal{E}=\emptyset$, which will be the only initial state, or the following conditions are satisfied:

(a) there is a unique extended sphere $(N, \lessdot, \mu, \lambda, \gamma, \alpha, \operatorname{col}) \in \mathcal{E}$ such that $\gamma=\alpha$ (we set $\operatorname{core}(\mathcal{E}):=(N, \lessdot, \mu, \lambda, \gamma))$

(b) there is $a \in \Sigma$ such that, for every $(N, \lessdot, \mu, \lambda, \gamma, \alpha, \operatorname{col}) \in \mathcal{E}, \lambda(\alpha)=a$

(so that we can assign a unique label $a$ to $\mathcal{E}$, denoted by $\operatorname{label}(\mathcal{E})$ )

(c) for every two elements $E=(N, \lessdot, \mu, \lambda, \gamma, \alpha, c o l)$ and $E^{\prime}=\left(N^{\prime}, \lessdot^{\prime}, \mu^{\prime}, \lambda^{\prime}, \gamma^{\prime}, \alpha^{\prime}, c o l^{\prime}\right)$ from $\mathcal{E}$, if $\operatorname{core}(E)=\operatorname{core}\left(E^{\prime}\right)$ and $\operatorname{col}=\operatorname{col}^{\prime}$, then $\alpha=\alpha^{\prime}$

So let us turn to the transition relation $\delta=\left\langle\delta_{1}, \delta_{2}\right\rangle$ :

- For $\mathcal{E}, \mathcal{E}^{\prime} \in Q$ and $a \in \Sigma$, we let $\left(\mathcal{E}, a, \mathcal{E}^{\prime}\right) \in \delta_{1}$ if $\mathcal{E}^{\prime} \neq \emptyset$ and the following hold:

(1) for all $(N, \lessdot, \mu, \lambda, \gamma, \alpha, c o l) \in \mathcal{E}^{\prime}, \alpha \notin \operatorname{dom}\left(\mu^{-1}\right)$ (i.e., $\mu^{-1}(\alpha)$ is not defined)

(2) $\operatorname{label}\left(\mathcal{E}^{\prime}\right)=a$

(3) for all $E=(N, \lessdot, \mu, \lambda, \gamma, \alpha, c o l) \in \mathcal{E}$ and $i \in N$,

$$
E[i] \in \mathcal{E}^{\prime} \Longrightarrow(\alpha, i) \in \lessdot
$$

(4) for all $E=(N, \lessdot, \mu, \lambda, \gamma, \alpha, c o l) \in \mathcal{E}^{\prime}$,

$$
\mathcal{E} \neq \emptyset \wedge \neg \exists i:(i, \alpha) \in \lessdot \Longrightarrow d_{E}(\gamma, \alpha)=r
$$

(5) for all $E=(N, \lessdot, \mu, \lambda, \gamma, \alpha, \operatorname{col}) \in \mathcal{E}$,

$$
\neg \exists i:(\alpha, i) \in \lessdot \Longrightarrow d_{E}(\gamma, \alpha)=r
$$

(6) for all $E=(N, \lessdot, \mu, \lambda, \gamma, \alpha, c o l) \in \mathcal{E}^{\prime}$ and $i \in N$,

$$
(i, \alpha) \in \lessdot \Longrightarrow E[i] \in \mathcal{E}
$$

(7) for all $E=(N, \lessdot, \mu, \lambda, \gamma, \alpha, c o l) \in \mathcal{E}$ and $i \in N$,

$$
(\alpha, i) \in \lessdot \Longrightarrow E[i] \in \mathcal{E}^{\prime}
$$

- For $\mathcal{E}_{c}, \mathcal{E}, \mathcal{E}^{\prime} \in Q$ and $a \in \Sigma_{r}$, we let $\left(\mathcal{E}_{c}, \mathcal{E}, a, \mathcal{E}^{\prime}\right) \in \delta_{2}$ if $\mathcal{E}_{c}, \mathcal{E}, \mathcal{E}^{\prime} \neq \emptyset$ and $(2)-(7)$ as above hold as well as the following:

(3') for all $E=(N, \lessdot, \mu, \lambda, \gamma, \alpha, c o l) \in \mathcal{E}_{c}$ and $i \in N$,

$$
E[i] \in \mathcal{E}^{\prime} \Longrightarrow(\alpha, i) \in \mu
$$

(4') for all $E=(N, \lessdot, \mu, \lambda, \gamma, \alpha, c o l) \in \mathcal{E}^{\prime}$,

$$
\alpha \notin \operatorname{dom}\left(\mu^{-1}\right) \Longrightarrow d_{E}(\gamma, \alpha)=r
$$

$\left(5^{\prime}\right)$ for all $E=(N, \lessdot, \mu, \lambda, \gamma, \alpha, c o l) \in \mathcal{E}_{c}$,

$$
\alpha \notin \operatorname{dom}(\mu) \Longrightarrow d_{E}(\gamma, \alpha)=r
$$

(6') for all $E=(N, \lessdot, \mu, \lambda, \gamma, \alpha, c o l) \in \mathcal{E}^{\prime}$,

$$
\alpha \in \operatorname{dom}\left(\mu^{-1}\right) \Longrightarrow E\left[\mu^{-1}(\alpha)\right] \in \mathcal{E}_{c}
$$

(7') for all $E=(N, \lessdot, \mu, \lambda, \gamma, \alpha, c o l) \in \mathcal{E}_{c}$,

$$
\alpha \in \operatorname{dom}(\mu) \Longrightarrow E[\mu(\alpha)] \in \mathcal{E}^{\prime}
$$

As already mentioned, the only initial state of $\mathcal{B}_{r}$ is the empty set, i.e., $Q_{I}=\{\emptyset\}$. Moreover, $\mathcal{E} \in Q$ is a final state if, for every extended sphere $(N, \lessdot, \mu, \lambda, \gamma, \alpha, c o l) \in \mathcal{E}$, both $\alpha \notin$ $\operatorname{dom}(\mu)$ and there is no $i \in N$ such that $(\alpha, i) \in \lessdot$. Finally, $\mathcal{E}$ is contained in $C$, the set of calling states, if there is $(N, \lessdot, \mu, \lambda, \gamma, \alpha, \operatorname{col}) \in \mathcal{E}$ such that $\alpha \in \operatorname{dom}(\mu)$. 
The mapping $\eta: Q \rightarrow$ Spheres $_{r}(\widetilde{\Sigma})$ as required in Proposition 4.1 is provided by core. More precisely, we set $\eta(\emptyset)$ to be some arbitrary sphere and $\eta(\mathcal{E})=\operatorname{core}(\mathcal{E})$ if $\mathcal{E} \neq \emptyset$.

Let us come back to the example in Figure 6, depicting two states, $\mathcal{E}$ and $\mathcal{E}^{\prime}$, of the sphere automaton for radius $r=1$, and a nested word that makes use of these states for being accepted. The sphere automaton contains a transition $\left(\mathcal{E}_{c}, \mathcal{E}, \bar{a}, \mathcal{E}^{\prime}\right)$ for some $\mathcal{E}_{c}$.

We will verify in the following that conditions (2)-(7) are indeed satisfied. The cases $\left(3^{\prime}\right)-\left(7^{\prime}\right)$ as well as the construction of $\mathcal{E}_{c}$ are left to the reader.

(2) All the active nodes in $\mathcal{E}^{\prime}$ are labeled with $\bar{a}$.

(3) Whenever a sphere from $\mathcal{E}$ is already present in $\mathcal{E}^{\prime}$, then the corresponding active nodes are in the $\lessdot$-relation. This applies to $E_{1}$ and $E_{1}^{\prime}$ as well as to $E_{2}$ and $E_{2}^{\prime}$.

(4) The extended sphere $E_{4}^{\prime}$ is the only one in $\mathcal{E}^{\prime}$ whose active node has no «-predecessor. However, the distance between this active node and the sphere center equals $r=1$.

(5) There is one extended sphere in $\mathcal{E}$ without a $\lessdot$-successor wrt. the active node, namely $E_{4}$. As required, the distance to the sphere center is $r=1$.

(6) There are three extended spheres in $\mathcal{E}^{\prime}$ whose active nodes have a «-predecessor: $E_{1}^{\prime}$, $E_{2}^{\prime}$, and $E_{3}^{\prime}$. In fact, $\mathcal{E}$ contains, in terms of $E_{1}, E_{2}$, and, respectively, $E_{3}$, all three extended spheres with the active node replaced by the respective $\lessdot$-predecessor.

(7) Symmetrically to the case $(6), E_{1}, E_{2}$, and $E_{3}$ from $\mathcal{E}$, where the active node is followed by a «-successor, have their counterparts in $\mathcal{E}^{\prime}$ in terms of $E_{1}^{\prime}, E_{2}^{\prime}$, and $E_{3}^{\prime}$, respectively.

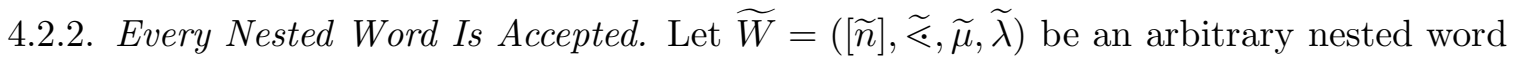
over $\widetilde{\Sigma}$. We show that $\widetilde{W} \in \mathcal{L}\left(\mathcal{B}_{r}\right)$. Let us first distribute colors to each of the involved spheres. For this, we define the notion of an overlap: for any $i, i^{\prime} \in[\widetilde{n}], i$ and $i^{\prime}$ are said to have an $r$-overlap in $\widetilde{W}$ if $r$-Sph $(\widetilde{W}, i) \cong r-\operatorname{Sph}\left(\widetilde{W}, i^{\prime}\right)$ and $d_{\widetilde{W}}\left(i, i^{\prime}\right) \leq 2 r+1$. For example, in Figure 5, $i$ and $i^{\prime}$ have a 2-overlap.

Claim 4.4. There is a mapping $\chi:[\widetilde{n}] \rightarrow[\# C o l]$ such that, for all $i, i^{\prime} \in[\widetilde{n}]$ with $i \neq i^{\prime}$, the following holds: if $i$ and $i^{\prime}$ have an $r$-overlap in $\widetilde{W}$, then $\chi(i) \neq \chi\left(i^{\prime}\right)$.

Proof. The mapping is obtained as a graph coloring. Consider the graph $([\widetilde{n}]$, Arcs $), \operatorname{Arcs} \subseteq$ $[\widetilde{n}] \times[\widetilde{n}]$, where, for $i, i^{\prime} \in[\widetilde{n}]$, we have $\left(i, i^{\prime}\right) \in \operatorname{Arcs}$ iff $i \neq i^{\prime}$ and $i$ and $i^{\prime}$ have an $r$-overlap in $\widetilde{W}$. Observe that $([\widetilde{n}], A r c s)$ cannot be of degree greater than $4 \cdot \operatorname{maxSize}(r)^{2}$. For each $i \in[\widetilde{n}]$, there are at most four distinct events $i^{\prime}$ such that $d_{\widetilde{W}}\left(i, i^{\prime}\right) \leq 1$. Now, if a position $j \in[\widetilde{n}]$ wants to "get in touch" with $i$, it requires a position in its own sphere, another position in the sphere around $i$, and one of the four possibilities to relate these two positions. Hence, $([\widetilde{n}]$, Arcs $)$ can be \#Col-colored by a mapping $\chi:[\widetilde{n}] \rightarrow[\# C o l]$ (i.e., $\chi(i) \neq \chi\left(i^{\prime}\right)$ for every $\left(i, i^{\prime}\right) \in$ Arcs $)$, which concludes the proof of Claim 4.4.

We now specify $\rho:[\widetilde{n}] \rightarrow Q:$ for $i \in[\widetilde{n}]$, we set $\rho(i)=\left\{\left(r-\operatorname{Sph}\left(\widetilde{W}, i^{\prime}\right), i, \chi\left(i^{\prime}\right)\right) \mid i^{\prime} \in[\widetilde{n}]\right.$ such that $\left.d_{\widetilde{W}}\left(i, i^{\prime}\right) \leq r\right\}$. With this definition, we can check that, for all $i \in[\widetilde{n}], \rho(i)$ is a valid state of $\mathcal{B}_{r}$, and that $\rho$ is indeed an accepting run of $\mathcal{B}_{r}$ on $\widetilde{W}$. So let $i \in[\widetilde{n}]$ and let $E=(N, \lessdot, \mu, \lambda, \gamma, \alpha, c o l)$ and $E^{\prime}=\left(N^{\prime}, \lessdot^{\prime}, \mu^{\prime}, \lambda^{\prime}, \gamma^{\prime}, \alpha^{\prime}, \operatorname{col}^{\prime}\right)$ be contained in $\rho(i)$.

(a) Assume that $\gamma=\alpha$ and $\gamma^{\prime}=\alpha^{\prime}$. Then, $(N, \lessdot, \mu, \lambda, \gamma, \gamma) \cong(r-\operatorname{Sph}(\widetilde{W}, i), i)$ and $\left(N^{\prime}, \lessdot^{\prime}, \mu^{\prime}, \lambda^{\prime}, \gamma^{\prime}, \gamma^{\prime}\right) \cong(r-\operatorname{Sph}(\widetilde{W}, i), i)$. Consequently, we have $(N, \lessdot, \mu, \lambda, \gamma, \gamma) \cong$ $\left(N^{\prime}, \lessdot^{\prime}, \mu^{\prime}, \lambda^{\prime}, \gamma^{\prime}, \gamma^{\prime}\right)$. Moreover, col $=\operatorname{col}^{\prime}=\chi(i)$.

(b) Of course, $\lambda(\alpha)=\lambda^{\prime}\left(\alpha^{\prime}\right)$. 
(c) Assume $(N, \lessdot, \mu, \lambda, \gamma) \cong\left(N^{\prime}, \lessdot^{\prime}, \mu^{\prime}, \lambda^{\prime}, \gamma^{\prime}\right)$ and col $=\operatorname{col}^{\prime}$. There are $i_{1}, i_{2} \in[\widetilde{n}]$ with $d_{\widetilde{W}}\left(i, i_{1}\right) \leq r, d_{\widetilde{W}}\left(i, i_{2}\right) \leq r,(N, \lessdot, \mu, \lambda, \gamma, \alpha) \cong\left(r-\operatorname{Sph}\left(\widetilde{W}, i_{1}\right), i\right),\left(N, \lessdot, \mu, \lambda, \gamma, \alpha^{\prime}\right) \cong$ $\left(r-\operatorname{Sph}\left(\widetilde{W}, i_{2}\right), i\right)$, and col $=\chi\left(i_{1}\right)=\chi\left(i_{2}\right)$. Clearly, we have $r-\operatorname{Sph}\left(\widetilde{W}, i_{1}\right) \cong r-\operatorname{Sph}\left(\widetilde{W}, i_{2}\right)$. Furthermore, $i_{1}=i_{2}$ and, therefore, $\alpha=\alpha^{\prime}$. This is because $i_{1}$ and $i_{2}$ have an $r$ overlap in $\widetilde{W}$ so that, according to Claim $4.4, i_{1} \neq i_{2}$ would imply $\chi\left(i_{1}\right) \neq \chi\left(i_{2}\right)$, which contradicts the premise.

Now, for $i \in\{0, \ldots, \widetilde{n}\}$ and $i^{\prime}=i+1$ with $i^{\prime} \notin \operatorname{dom}\left(\widetilde{\mu}^{-1}\right)$, we check that the triple $\left(\rho(i), \lambda\left(i^{\prime}\right), \rho\left(i^{\prime}\right)\right)$ is contained in $\delta_{1}$, where we let $\rho(0)=\emptyset$. Note first that, of course, $\rho\left(i^{\prime}\right) \neq \emptyset$.

(1) Suppose $E=(N, \lessdot, \mu, \lambda, \gamma, \alpha, c o l) \in \rho\left(i^{\prime}\right)$. We have $E \cong\left(r-\operatorname{Sph}\left(\widetilde{W}, i^{\prime \prime}\right), i^{\prime}, \chi\left(i^{\prime \prime}\right)\right)$ for some $i^{\prime \prime} \in[\widetilde{n}]$ with $d_{\widetilde{W}}\left(i^{\prime}, i^{\prime \prime}\right) \leq r$. As $i^{\prime} \notin \operatorname{dom}\left(\widetilde{\mu}^{-1}\right)$, we deduce $\alpha \notin \operatorname{dom}\left(\mu^{-1}\right)$.

(2) Obviously, we have label $\left(\rho\left(i^{\prime}\right)\right)=\widetilde{\lambda}\left(i^{\prime}\right)$.

(3) Suppose $E=(N, \lessdot, \mu, \lambda, \gamma, \alpha, c o l) \in \rho(i)$ (we thus have $i \geq 1$ ) and $j \in N$ such that $E[j] \in \rho\left(i^{\prime}\right)$. Recall that we have to show that, then, $(\alpha, j) \in \lessdot$. There are $i_{1}, i_{1}^{\prime} \in[\widetilde{n}]$ such that $d_{\widetilde{W}}\left(i_{1}, i\right) \leq r, d_{\widetilde{W}}\left(i_{1}^{\prime}, i^{\prime}\right) \leq r,(N, \lessdot, \mu, \lambda, \gamma, \alpha) \cong\left(r-\operatorname{Sph}\left(\widetilde{W}, i_{1}\right), i\right)$, $(N, \lessdot, \mu, \lambda, \gamma, j) \cong\left(r-\operatorname{Sph}\left(\widetilde{W}, i_{1}^{\prime}\right), i^{\prime}\right)$, and $\operatorname{col}=\chi\left(i_{1}\right)=\chi\left(i_{1}^{\prime}\right)$. We easily see that $i_{1}$ and $i_{1}^{\prime}$ have an $r$-overlap in $\widetilde{W}$. We deduce, according to Claim 4.4. $i_{1}=i_{1}^{\prime}$. As, then, $(N, \lessdot, \mu, \lambda, \gamma, \alpha) \cong\left(r-\operatorname{Sph}\left(\widetilde{W}, i_{1}\right), i\right),(N, \lessdot, \mu, \lambda, \gamma, j) \cong\left(r-\operatorname{Sph}\left(\widetilde{W}, i_{1}\right), i^{\prime}\right)$, and $\left(i, i^{\prime}\right) \in \widetilde{\varkappa}$, we can infer $(\alpha, j) \in \lessdot$.

(4) Let $E=(N, \lessdot, \mu, \lambda, \gamma, \alpha, c o l) \in \rho\left(i^{\prime}\right)$, suppose $i^{\prime} \geq 2$, and suppose that there is no $j \in N$ such that $(j, \alpha) \in \lessdot$. Recall that we have to show that $d_{E}(\gamma, \alpha)=r$. There is $i_{1}^{\prime} \in[\widetilde{n}]$ such that $d_{\widetilde{W}}\left(i_{1}^{\prime}, i^{\prime}\right) \leq r$ and $(N, \lessdot, \mu, \lambda, \gamma, \alpha) \cong\left(r-\operatorname{Sph}\left(\widetilde{W}, i_{1}^{\prime}\right), i^{\prime}\right)$. But if $d_{E}(\gamma, \alpha)<r$, then $d_{\widetilde{W}}\left(i_{1}^{\prime}, i^{\prime}\right)<r$, and there must be a $\lessdot$-predecessor of $\alpha$, which is a contradiction. We therefore deduce that $d_{E}(\gamma, \alpha)=r$.

(5) Let $E=(N, \lessdot, \mu, \lambda, \gamma, \alpha, c o l) \in \rho(i)$ and suppose that there is no $j \in N$ such that $(\alpha, j) \in \lessdot$. Similarly to the case $(4)$, we show that $d_{E}(\gamma, \alpha)=r$. In fact, there is $i_{1} \in[\widetilde{n}]$ such that $d_{\widetilde{W}}\left(i_{1}, i\right) \leq r$ and $(N, \lessdot, \mu, \lambda, \gamma, \alpha) \cong\left(r-\operatorname{Sph}\left(\widetilde{W}, i_{1}\right), i\right)$. Again, if $d_{E}(\gamma, \alpha)<r$, then $d_{\widetilde{W}}\left(i_{1}, i\right)<r$ so that there must be a $\lessdot$-successor of $\alpha$, which is a contradiction. We conclude that $d_{E}(\gamma, \alpha)=r$.

(6) Let $E=(N, \lessdot, \mu, \lambda, \gamma, \alpha, c o l) \in \rho\left(i^{\prime}\right)$ and $j \in N$ such that $(j, \alpha) \in \lessdot$. We show that, then, $E[j] \in \rho(i)$. There is $i_{1}^{\prime} \in[\widetilde{n}]$ such that $d_{\widetilde{W}}\left(i_{1}^{\prime}, i^{\prime}\right) \leq r,(N, \lessdot, \mu, \lambda, \gamma, \alpha) \cong$ $\left(r-\operatorname{Sph}\left(\widetilde{W}, i_{1}^{\prime}\right), i^{\prime}\right)$, and $\operatorname{col}=\chi\left(i_{1}^{\prime}\right)$. As $(j, \alpha) \in \lessdot, \alpha$ is not minimal so that we have $i \geq 1$. Since, furthermore, $d_{E}(\gamma, j) \leq r$ implies $d_{\widetilde{W}}\left(i_{1}^{\prime}, i\right) \leq r$, and since we also have $(N, \lessdot, \mu, \lambda, \gamma, j) \cong\left(r-\operatorname{Sph}\left(\widetilde{W}, i_{1}^{\prime}\right), i\right)$ and $\operatorname{col}=\chi\left(i_{1}^{\prime}\right)$, we deduce $E[j]=$ $(N, \lessdot, \mu, \lambda, \gamma, j, \operatorname{col}) \in \rho(i)$.

(7) Let $E=(N, \lessdot, \mu, \lambda, \gamma, \alpha, c o l) \in \rho(i)$ and $j \in N$ such that $(\alpha, j) \in \lessdot$. We have to show that $E[j] \in \rho\left(i^{\prime}\right)$. There is $i_{1} \in[\widetilde{n}]$ such that $d_{\widetilde{W}}\left(i_{1}, i\right) \leq r,(N, \lessdot, \mu, \lambda, \gamma, \alpha) \cong$ $\left(r-\operatorname{Sph}\left(\widetilde{W}, i_{1}\right), i\right)$, and $\operatorname{col}=\chi\left(i_{1}\right)$. Since $d_{E}(\gamma, j) \leq r$ implies $d_{\widetilde{W}}\left(i_{1}, i^{\prime}\right) \leq r$, and since we have $(N, \lessdot, \mu, \lambda, \gamma, j) \cong\left(r-\operatorname{Sph}\left(\widetilde{W}, i_{1}\right), i^{\prime}\right)$ and $\operatorname{col}=\chi\left(i_{1}\right)$, we deduce $E[j]=$ $(N, \lessdot, \mu, \lambda, \gamma, j, \mathrm{col}) \in \rho\left(i^{\prime}\right)$.

Next, for $i_{c}, i, i^{\prime} \in[\widetilde{n}]$ with $i^{\prime}=i+1$ and $\left(i_{c}, i^{\prime}\right) \in \widetilde{\mu}$, we check that the quadruple $\left(\rho\left(i_{c}\right), \rho(i), \lambda\left(i^{\prime}\right), \rho\left(i^{\prime}\right)\right)$ is contained in $\delta_{2}$. Checking (2)-(7) proceeds as in the above cases. 
For completeness, we present the cases $\left(3^{\prime}\right)-\left(7^{\prime}\right)$, which are shown analogously. First observe that, indeed, $\rho\left(i_{c}\right), \rho(i)$, and $\rho\left(i^{\prime}\right)$ are all nonempty.

(3') Suppose $E=(N, \lessdot, \mu, \lambda, \gamma, \alpha, c o l) \in \rho\left(i_{c}\right)$ and $j \in N$ such that $E[j] \in \rho\left(i^{\prime}\right)$. We show that $(\alpha, j) \in \mu$. There are $i_{1}, i_{1}^{\prime} \in[\widetilde{n}]$ such that $d_{\widetilde{W}}\left(i_{1}, i_{c}\right) \leq r, d_{\widetilde{W}}\left(i_{1}^{\prime}, i^{\prime}\right) \leq r$, $(N, \lessdot, \mu, \lambda, \gamma, \alpha) \cong\left(r-\operatorname{Sph}\left(\widetilde{W}, i_{1}\right), i_{c}\right),(N, \lessdot, \mu, \lambda, \gamma, j) \cong\left(r-\operatorname{Sph}\left(\widetilde{W}, i_{1}^{\prime}\right), i^{\prime}\right)$, and $c o l=$ $\chi\left(i_{1}\right)=\chi\left(i_{1}^{\prime}\right)$. Again, $i_{1}$ and $i_{1}^{\prime}$ have an $r$-overlap in $\widetilde{W}$. According to Claim 4.4, $i_{1}=i_{1}^{\prime}$. Then, $(N, \lessdot, \mu, \lambda, \gamma, \alpha) \cong\left(r-\operatorname{Sph}\left(\widetilde{W}, i_{1}\right), i_{c}\right),(N, \lessdot, \mu, \lambda, \gamma, j) \cong\left(r-\operatorname{Sph}\left(\widetilde{W}, i_{1}\right), i^{\prime}\right)$, and $\left(i_{c}, i^{\prime}\right) \in \widetilde{\mu}$, so that we can deduce $(\alpha, j) \in \mu$.

(4') Let $E=(N, \lessdot, \mu, \lambda, \gamma, \alpha, c o l) \in \rho\left(i^{\prime}\right)$ and suppose that there is no $j \in N$ such that $(j, \alpha) \in \mu$. We have to show that $d_{E}(\gamma, \alpha)=r$. There is $i_{1}^{\prime} \in[\widetilde{n}]$ such that $d_{\widetilde{W}}\left(i_{1}^{\prime}, i^{\prime}\right) \leq r$ and $(N, \lessdot, \mu, \lambda, \gamma, \alpha) \cong\left(r-\operatorname{Sph}\left(\widetilde{W}, i_{1}^{\prime}\right), i^{\prime}\right)$. But if $d_{E}(\gamma, \alpha)<r$, then $d_{\widetilde{W}}\left(i_{1}^{\prime}, i^{\prime}\right)<r$, so there must be a $\mu$-predecessor of $\alpha$, which is a contradiction. We deduce $d_{E}(\gamma, \alpha)=r$.

(5') Let $E=(N, \lessdot, \mu, \lambda, \gamma, \alpha, c o l) \in \rho\left(i_{c}\right)$ and suppose that there is no $j \in N$ such that $(\alpha, j) \in \mu$. We show that, then, $d_{E}(\gamma, \alpha)=r$. There is $i_{1} \in[\widetilde{n}]$ such that $d_{\widetilde{W}}\left(i_{1}, i_{c}\right) \leq r$ and $(N, \lessdot, \mu, \lambda, \gamma, \alpha) \cong\left(r-\operatorname{Sph}\left(\widetilde{W}, i_{1}\right), i_{c}\right)$. If $d_{E}(\gamma, \alpha)<r$, then $d_{\widetilde{W}}\left(i_{1}, i_{c}\right)<r$, so there must be a $\mu$-successor of $\alpha$, which is a contradiction. We conclude that $d_{E}(\gamma, \alpha)=r$.

(6') Let $E=(N, \lessdot, \mu, \lambda, \gamma, \alpha, c o l) \in \rho\left(i^{\prime}\right)$ and $j \in N$ such that $(j, \alpha) \in \mu$. We show $E[j] \in$ $\rho\left(i_{c}\right)$. There is $i_{1}^{\prime} \in[\widetilde{n}]$ such that $d_{\widetilde{W}}\left(i_{1}^{\prime}, i^{\prime}\right) \leq r,(N, \lessdot, \mu, \lambda, \gamma, \alpha) \cong\left(r-\operatorname{Sph}\left(\widetilde{W}, i_{1}^{\prime}\right), i^{\prime}\right)$, and col $=\chi\left(i_{1}^{\prime}\right)$. Due to $d_{E}(\gamma, j) \leq r$, we also have $d_{\widetilde{W}}\left(i_{1}^{\prime}, i_{c}\right) \leq r$, and since $(N, \lessdot, \mu, \lambda, \gamma, j) \cong\left(r-\operatorname{Sph}\left(\widetilde{W}, i_{1}^{\prime}\right), i_{c}\right)$ and $c o l=\chi\left(i_{1}^{\prime}\right)$, we deduce $E[j] \in \rho\left(i_{c}\right)$.

(7') Let $E=(N, \lessdot, \mu, \lambda, \gamma, \alpha, c o l) \in \rho\left(i_{c}\right)$ and $j \in N$ such that $(\alpha, j) \in \mu$. We have to show $E[j] \in \rho\left(i^{\prime}\right)$. There is $i_{1} \in[\widetilde{n}]$ such that $d_{\widetilde{W}}\left(i_{1}, i_{c}\right) \leq r,(N, \lessdot, \mu, \lambda, \gamma, \alpha) \cong$ $\left(r-\operatorname{Sph}\left(\widetilde{W}, i_{1}\right), i_{c}\right)$, and col $=\chi\left(i_{1}\right)$. From $d_{E}(\gamma, j) \leq r$, it follows $d_{\widetilde{W}}\left(i_{1}, i^{\prime}\right) \leq r$. As, moreover, $(N, \lessdot, \mu, \lambda, \gamma, j) \cong\left(r-\operatorname{Sph}\left(\widetilde{W}, i_{1}\right), i^{\prime}\right)$ and $\operatorname{col}=\chi\left(i_{1}\right)$, we deduce $E[j]=$ $(N, \lessdot, \mu, \lambda, \gamma, j, \mathrm{col}) \in \rho\left(i^{\prime}\right)$.

4.2.3. Every Run Keeps Track Of Spheres. We will now show that an accepting run reveals the sphere around any node. This constitutes the more difficult part of the correctness proof.

We introduce some useful notation: By $\Delta$, we denote the set $\{\rightarrow, \leftarrow, \curvearrowright, \curvearrowleft, \cup, \smile\}$ of directions. Now let $W=([n], \lessdot, \mu, \lambda) \in \mathbb{N} \mathbb{W}(\widetilde{\Sigma})$ be a nested word, $i, j \in[n]$, and let $w=e_{1} \ldots e_{m} \in \Delta^{*}$ (where $e_{k} \in \Delta$ for all $k \in\{1, \ldots, m\}$ ). We write $i \stackrel{w}{\Longrightarrow} w j$ if there are $i_{0}, i_{1}, \ldots, i_{m} \in[n]$ such that $i_{0}=i, i_{m}=j$, and, for every $k \in\{0, \ldots, m-1\}$, one of the following holds:

(a) $e_{k+1}=\rightarrow$ and $i_{k+1}=i_{k}+1$

(b) $e_{k+1}=\leftarrow$ and $i_{k+1}=i_{k}-1$

(c) $e_{k+1}=\curvearrowright$ and $i_{k} \in \operatorname{dom}(\mu)$ and $\lambda\left(i_{k}\right) \in \Sigma_{c}^{1}$ and $i_{k+1}=\mu\left(i_{k}\right)$

(d) $e_{k+1}=\cup$ and $i_{k} \in \operatorname{dom}(\mu)$ and $\lambda\left(i_{k}\right) \in \Sigma_{c}^{2}$ and $i_{k+1}=\mu\left(i_{k}\right)$

(e) $e_{k+1}=\curvearrowleft$ and $i_{k} \in \operatorname{dom}\left(\mu^{-1}\right)$ and $\lambda\left(i_{k}\right) \in \Sigma_{r}^{1}$, and $i_{k+1}=\mu^{-1}\left(i_{k}\right)$

(f) $e_{k+1}=\smile$ and $i_{k} \in \operatorname{dom}\left(\mu^{-1}\right)$ and $\lambda\left(i_{k}\right) \in \Sigma_{r}^{2}$, and $i_{k+1}=\mu^{-1}\left(i_{k}\right)$

Moreover, we write $i \stackrel{w}{\longleftrightarrow} W j$ if there are pairwise distinct $i_{0}, i_{1}, \ldots, i_{m-1} \in[n]$ and $i_{m} \in$ $[n] \backslash\left\{i_{1}, \ldots, i_{m-1}\right\}$ such that $i_{0}=i, i_{m}=j$, and, for every $k \in\{0, \ldots, m-1\}$, (a)-(f) as above hold. 
We say that a string $w \in \Delta^{+}$is circular if $i \stackrel{w}{\longleftrightarrow} W i$ for some nested word $W \in \mathbb{N} \mathbb{W}(\widetilde{\Sigma})$ and some position $i$ of $W$. In other words, a circular string can produce a circle in a nested word. For example, $\curvearrowright \rightarrow \smile \rightarrow$ and $\curvearrowright \rightarrow \hookrightarrow \rightarrow \curvearrowleft \rightarrow$ are circular (for an appropriate alphabet $\widetilde{\Sigma})$, whereas $\curvearrowright \rightarrow \cup \rightarrow \curvearrowleft \leftarrow$ is not circular.

The following proposition is crucial for our project, and it fails when considering nested words over more than two stacks.

Proposition 4.5. Let $w \in \Delta^{+}$be circular. Then, for all $k \geq 2$, $w^{k}$ is not circular.

Before we prove Proposition 4.5, observe that it does not hold as soon as a third stack comes into play. To see this, consider Figure 7 describing a part of a nested word $W$ over the 3 -stack call-return alphabet $\langle\{(\{a\},\{\bar{a}\}),(\{b\},\{\bar{b}\}),(\{c\},\{\bar{c}\})\}, \emptyset\rangle$. Suppose $w=\curvearrowright \leftarrow \cup \leftarrow \stackrel{3}{n} \leftarrow$ (where the meaning of $\stackrel{3}{n}$ is the expected one), which is circular if we apply our definition to the framework of three stacks. However, we have $i \stackrel{w w}{\longrightarrow} W$. It should be noted that this does not imply that there is no sphere automaton or logical characterization in the framework with more than two stacks. Indeed, we leave as an open question if multiple stacks generally allow for a logical characterization in terms of a fragment of MSO logic.

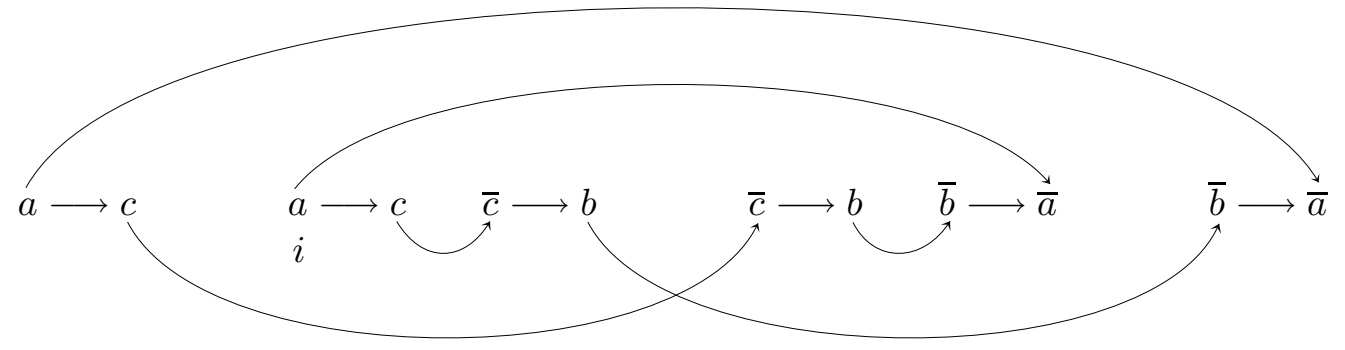

Figure 7: Proposition 4.5 fails when considering three stacks

In the above definition of $i \stackrel{w}{\longrightarrow} W j$, it is crucial to require the elements $i_{0}, i_{1}, \ldots, i_{m-1} \in$ $[n]$ to be pairwise distinct. This can be seen considering a part of the nested word $W$ over the 2-stack call-return alphabet $\langle\{(\{a\},\{\bar{a}\}),(\{b\},\{\bar{b}\})\}, \emptyset\rangle$ that is depicted in Figure 8 , Let

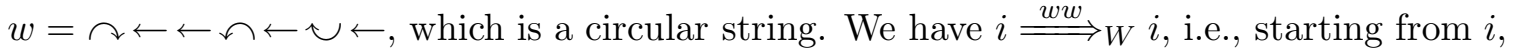
we can follow the sequence of directions $w$ twice, arriving at $i$ again. However, apart from $i$, we have to visit $j_{1}$ and $j_{2}$ twice. Indeed, $i \stackrel{w w}{\longleftrightarrow} i$.

Proof. (of Proposition [4.5). Let $W=([n], \lessdot, \mu, \lambda) \in \mathbb{N W}(\widetilde{\Sigma}), w \in \Delta^{+}$, and $i \in[n]$. We have to show that, if $i \stackrel{w}{\longleftrightarrow} W$, then $w$ cannot be decomposed nontrivially into identical circular factors, i.e., there is no circular $u \in \Delta^{+}$such that $w=u^{k}$ for some $k \geq 2$.

To see this easily, we observe that a situation such as $i \stackrel{w}{\longleftrightarrow} W i$ corresponds to a topological circle, as depicted in Figure 9. A topological circle is a closed line in the two-dimensional plane that never crosses over itself. Let us construct topological circles according to the following procedure: We assume a straight (horizontal) line of the plane.

\footnotetext{
${ }^{4}$ Actually, one can even show that there is no $u \in \Delta^{+}$at all (not even non-circular) such that $w=u^{k}$ for some $k \geq 2$.
} 


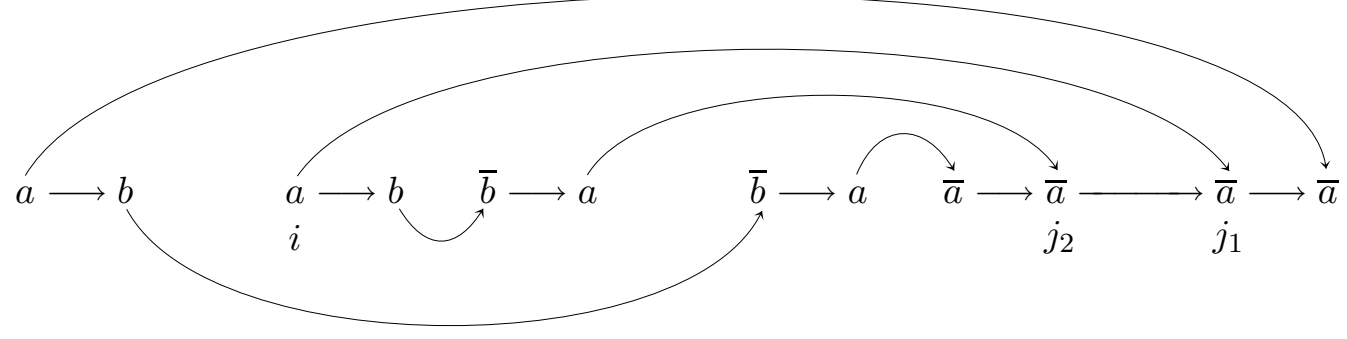

Figure 8: Intermediate positions need to be pairwise distinct

Assume further a point $i$ on this line. Starting from $i$, we choose another two points as follows: Pick a symbol $\gamma$ from the alphabet $\{\sim, \sim, \curvearrowright, G\}$. According to this choice, we first draw a semicircle above the straight line ending somewhere on the line, and then, without interruption, a semicircle below the line, again resulting in a point on the line. Each semicircle is drawn in the direction indicated by $\gamma$, e.g., $\bigcirc$ requires to draw the upper semicircle rightwards and the lower one leftwards, and $\leadsto$ requires both the upper and the lower semicircle to be drawn rightwards. This procedure is continued until we reach the original point $i$. We call a sequence from $\{\leadsto, \sim, \curvearrowright, G\}^{+}$that allows us to draw a topological circle circular. For example, in Figure 9, we construct a topological circle by following the sequence $x=\leadsto \sim \backsim \curvearrowright \backsim \sim$, starting in the left outermost point of intersection on the horizontal line. Thus, $x$ is circular, whereas $G D$ is not circular. Observe that we have $x \neq y^{k}$ for all $y \in\{\sim, \sim, \diamond, G\}^{+}$and $k \geq 2$.

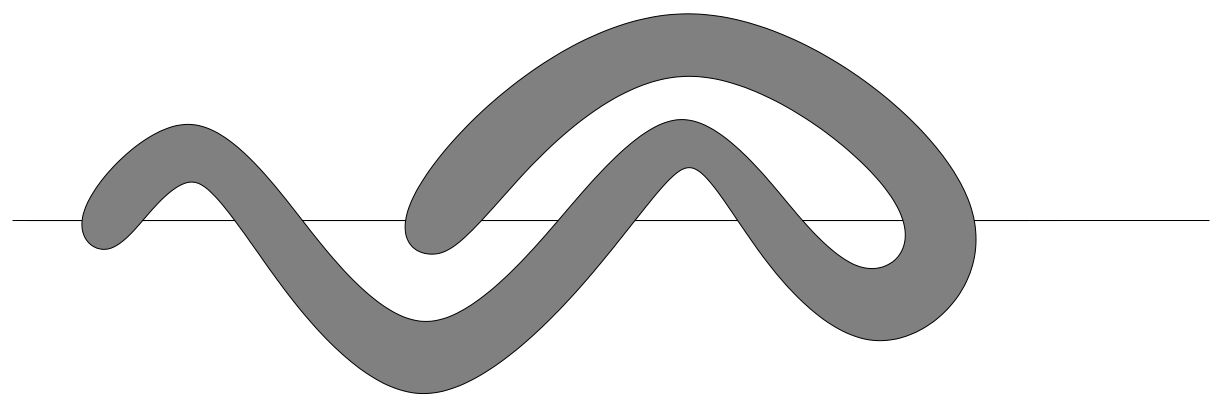

Figure 9: Proof of Proposition 4.5

It is not hard to see that topological circles behave aperiodically in general, i.e., for any given $y \in\{\sim, \sim, \supset, G\}$, there is no $k \geq 2$ such that $y^{k}$ is circular. To show our proposition, we can even restrict to circular $y$. So let $y \in\{\leadsto, \sim, \odot, G\}^{+}$. But if $y$ is circular, then, for growing $k, y^{k}$ gives rise to a "spiral", and going back to the starting point would require to intersect the line that has been drawn hitherto.

Let us relate our topological circles to the nested-word setting over two stacks. To this aim, we define a partial mapping $f: \Delta^{+} \rightarrow\{\sim, \sim, \supset, G\}^{+}$that associates with any circular string a sequence over $\{\leadsto, \sim, \ominus, G\}$. This is done by reading a string from left to right and successively replacing every direction from $\Delta$ with a symbol from $\{\sim, \sim, \curvearrowright, G\}$, according to the following rules: 
- $\curvearrowright$ is always replaced with $\leadsto$

- $\curvearrowleft$ is always replaced with $\sim$

- $\rightarrow$ is replaced with $\begin{cases}a & \text { if the previous letter has been } \curvearrowleft \\ \leadsto & \text { otherwise }\end{cases}$

- $\leftarrow$ is replaced with $\begin{cases}\circlearrowright & \text { if the previous letter has been } \curvearrowright \\ \sim & \text { otherwise }\end{cases}$

- $\checkmark$ is replaced with $\begin{cases}a & \text { if the previous letter has been } \leftarrow \\ \sim & \text { otherwise }\end{cases}$

- $\sim$ is replaced with $\begin{cases}\circlearrowright & \text { if the previous letter has been } \rightarrow \\ \sim & \text { otherwise }\end{cases}$

For example, $f(\leftarrow \curvearrowleft \rightarrow \cup \rightarrow \curvearrowleft \rightarrow \smile)=\backsim \backsim G \leadsto \leadsto \backsim G \curvearrowright$. Let $w$ be circular. Clearly, $f(w)$ is circular as well, i.e., it allows us to draw a topological circle. We assume that the first letter of $w$ stems from $\{\curvearrowright, \curvearrowleft\}$. Other cases are either trivial or can be reduced to that one. Then, if $w$ can be decomposed nontrivially into identical circular factors, then this also applies to $f(w)$. Summarizing, the power of a circular string is not circular anymore.

This concludes the proof of Proposition 4.5.

We will now show that, indeed, $\mathcal{B}_{r}$ discovers the $r$-sphere around any node of an input nested word.

Let $W=([n], \lessdot, \mu, \lambda) \in \mathbb{N} \mathbb{W}(\widetilde{\Sigma})$ be a nested word and $\rho$ be a run of $\mathcal{B}_{r}$ on $W$. Consider any $i \in[n]$, let $\left(N_{i}, \lessdot_{i}, \mu_{i}, \lambda_{i}, \gamma_{i}\right)$ refer to $\operatorname{core}(\rho(i))$, and let $\operatorname{col}_{i}$ be the unique element from [\#Col] satisfying $E_{i}:=\left(N_{i}, \lessdot_{i}, \mu_{i}, \lambda_{i}, \gamma_{i}, \gamma_{i}, \operatorname{col}_{i}\right) \in \rho(i)$.

The following statement claims that an arbitrarily long path in $E_{i}$ is simulated by a corresponding path in $W$.

Claim 4.6. Let $d \geq 0$ and suppose there are $j_{0}, \ldots, j_{d} \in N_{i}$ such that $\gamma_{i}=j_{0} \leftrightarrow E_{i} j_{1} \leftrightarrow E_{i}$ $\ldots \leftrightarrow E_{i} j_{d}$. Then, there is a (unique) sequence of nodes $i_{0}, \ldots, i_{d} \in[n]$ such that

- $i_{0}=i$,

- for each $k \in\{0, \ldots, d\}, E_{i}\left[j_{k}\right] \in \rho\left(i_{k}\right)$ (in particular, $\lambda\left(i_{k}\right)=\lambda_{i}\left(j_{k}\right)$ ), and

- for each $k \in\{0, \ldots, d-1\},\left(j_{k}, j_{k+1}\right) \sqsubseteq_{W}^{E_{i}}\left(i_{k}, i_{k+1}\right)$.

Proof. The proof is by induction. Obviously, the statement holds for $d=0$. So assume $d \geq 0$ and suppose there are a sequence $j_{0}, \ldots, j_{d}, j_{d+1} \in N_{i}$ such that $\gamma_{i}=j_{0} \leftrightarrow E_{i} j_{1} \leftrightarrow E_{i}$ $\ldots \leftrightarrow_{E_{i}} j_{d} \leftrightarrow_{E_{i}} j_{d+1}$ and a unique sequence $i_{0}, i_{1}, \ldots, i_{d} \in[n]$ such that $i_{0}=i, E_{i}\left[j_{k}\right] \in \rho(i)$ for each $k \in\{0, \ldots, d\}$, and $\left(j_{k}, j_{k+1}\right) \sqsubseteq_{W}^{E_{i}}\left(i_{k}, i_{k+1}\right)$ for each $k \in\{0, \ldots, d-1\}$. We consider four cases:

- Assume $\left(j_{d}, j_{d+1}\right) \in \lessdot_{i}$. Then, $\rho\left(i_{d}\right)$ is not a final state so that $i_{d}<n$. We set $i_{d+1}=i_{d}+1$. Due to $(7)$, we have $E_{i}\left[j_{d+1}\right] \in \rho\left(i_{d+1}\right)$.

- Assume $\left(j_{d+1}, j_{d}\right) \in \lessdot_{i}$. Then, according to (6), $i_{d} \geq 2$. We set $i_{d+1}=i_{d}-1$. Due to (6), we also have $E_{i}\left[j_{d+1}\right] \in \rho\left(i_{d+1}\right)$.

- Assume $\left(j_{d}, j_{d+1}\right) \in \mu_{i}$. Clearly, $\rho\left(i_{d}\right)$ is a calling state so that $\mu\left(i_{d}\right)$ is defined. Setting $i_{d+1}=\mu\left(i_{d}\right)$, we have, due to $\left(7^{\prime}\right), E_{i}\left[j_{d+1}\right] \in \rho\left(i_{d+1}\right)$.

- Assume $\left(j_{d+1}, j_{d}\right) \in \mu_{i}$. According to $(1), i_{d} \in \operatorname{dom}\left(\mu^{-1}\right)$. With (6'), letting $i_{d+1}=$ $\mu^{-1}\left(i_{d}\right)$, we have $E_{i}\left[j_{d+1}\right] \in \rho\left(i_{d+1}\right)$.

This concludes the proof of Claim 4.6. 
Claim 4.7. There is a homomorphism $h: r-\operatorname{Sph}(W, i) \rightarrow \operatorname{core}(\rho(i))$.

Proof. We show by induction the following statement:

For every $d \in\{0, \ldots, r\}$, there is a homomorphism $h: d-\operatorname{Sph}(W, i) \rightarrow$ $d$-Sph $\left(\left(N_{i}, \lessdot_{i}, \mu_{i}, \lambda_{i}\right), \gamma_{i}\right)$ such that, for each $i^{\prime} \in[n]$ with $d_{W}\left(i, i^{\prime}\right) \leq d$, we have $E_{i}\left[h\left(i^{\prime}\right)\right] \in \rho\left(i^{\prime}\right)$.

Of course, $(*)$ holds for $d=0$. So assume that $(*)$ holds true for some natural number $d \in\{0, \ldots, r-1\}$, i.e., there is a homomorphism $h: d-\operatorname{Sph}(W, i) \rightarrow d-\operatorname{Sph}\left(\left(N_{i}, \lessdot_{i}, \mu_{i}, \lambda_{i}\right), \gamma_{i}\right)$ such that $E_{i}\left[h\left(i^{\prime}\right)\right] \in \rho\left(i^{\prime}\right)$ for each $i^{\prime} \in[n]$ with $d_{W}\left(i, i^{\prime}\right) \leq d$. We show that then $\left(^{*}\right)$ holds for $d+1$ as well. For this, let $i_{1}, i_{2} \in[n]$ such that $d_{W}\left(i, i_{1}\right)=d$ and $d_{W}\left(i, i_{2}\right)=d+1$.

- Suppose $i_{1} \lessdot i_{2}$. Since $d_{W}\left(i, i_{1}\right)<r$, we also have $d_{E_{i}}\left(\gamma_{i}, h\left(i_{1}\right)\right)<r$. Due to $(5)$, there is $j_{2} \in N_{i}$ such that $h\left(i_{1}\right) \lessdot_{i} j_{2}$. Since $E_{i}\left[h\left(i_{1}\right)\right] \in \rho\left(i_{1}\right)$, we obtain, by $(7)$ and $(2)$, that $\lambda_{i}\left(j_{2}\right)=\lambda\left(i_{2}\right)$ and $E_{i}\left[j_{2}\right] \in \rho\left(i_{2}\right)$.

- Similarly, we proceed if $i_{2} \lessdot i_{1}$. By $d_{E_{i}}\left(\gamma_{i}, h\left(i_{1}\right)\right)<r$ and (4), there is $j_{2} \in N_{i}$ such that $j_{2} \lessdot_{i} h\left(i_{1}\right)$. Since $E_{i}\left[h\left(i_{1}\right)\right] \in \rho\left(i_{1}\right)$, we obtain, by $(6)$ and $(2)$, that $\lambda_{i}\left(j_{2}\right)=\lambda\left(i_{2}\right)$ and $E_{i}\left[j_{2}\right] \in \rho\left(i_{2}\right)$.

- If $\left(i_{1}, i_{2}\right) \in \mu$, then there exists, exploiting ( $\left.5^{\prime}\right)$ and $\left(7^{\prime}\right), j_{2} \in N_{i}$ such that $\left(h\left(i_{1}\right), j_{2}\right) \in \mu_{i}$, $\lambda_{i}\left(j_{2}\right)=\lambda\left(i_{2}\right)$, and $E_{i}\left[j_{2}\right] \in \rho\left(i_{2}\right)$.

- If $\left(i_{2}, i_{1}\right) \in \mu$, then we can find, due to (4') and (6'), $j_{2} \in N_{i}$ such that $\left(j_{2}, h\left(i_{1}\right)\right) \in \mu_{i}$, $\lambda_{i}\left(j_{2}\right)=\lambda\left(i_{2}\right)$, and $E_{i}\left[j_{2}\right] \in \rho\left(i_{2}\right)$.

Observe that $j_{2}$ is uniquely determined by $i_{2}$ and does not depend on the choice of $i_{1}$ or on the relation between $i_{1}$ and $i_{2}$ : If we obtained distinct elements $j_{2}$ and $j_{2}^{\prime}$, then the constraints $E_{i}\left[j_{2}\right] \in \rho\left(i_{2}\right)$ and $E_{i}\left[j_{2}^{\prime}\right] \in \rho\left(i_{2}\right)$ would imply that $\rho\left(i_{2}\right)$ is not a valid state.

The above procedure extends the domain of the homomorphism $h$ by those elements whose distance to $i$ is $d+1$. I.e., for $i_{1}, i_{2} \in[n]$ with $d_{W}\left(i, i_{1}\right)=d_{W}\left(i, i_{2}\right)=d+1$, we determined two unique elements $h\left(i_{1}\right), h\left(i_{2}\right) \in N_{i}$, respectively. Let us show that $\left(i_{1}, i_{2}\right) \sqsubseteq_{\text {core }(\rho(i))}^{W}\left(h\left(i_{1}\right), h\left(i_{2}\right)\right)$. Suppose $i_{1} \lessdot i_{2}$ (the case $i_{2} \lessdot i_{1}$ is symmetric). As $E_{i}\left[h\left(i_{1}\right)\right] \in \rho\left(i_{1}\right)$ and $E_{i}\left[h\left(i_{2}\right)\right] \in \rho\left(i_{2}\right)$, we have, by $(3), h\left(i_{1}\right) \lessdot_{i} h\left(i_{2}\right)$. Similarly, with $\left(3^{\prime}\right)$, $\left(i_{1}, i_{2}\right) \in \mu$ implies $\left(h\left(i_{1}\right), h\left(i_{2}\right)\right) \in \mu_{i}$.

Claim 4.8. There is a homomorphism $h^{\prime}: \operatorname{core}(\rho(i)) \rightarrow r$-Sph $(W, i)$.

Proof. We show, again by induction, the following statement:

For every natural number $d \in\{0, \ldots, r\}$, there is a homomorphism $h^{\prime}$ : $d$-Sph $\left(\left(N_{i}, \lessdot_{i}, \mu_{i}, \lambda_{i}\right), \gamma_{i}\right) \rightarrow d-\operatorname{Sph}(W, i)$ such that, for every $j \in N_{i}$ with $d_{E_{i}}\left(\gamma_{i}, j\right) \leq d$, we have $E_{i}[j] \in \rho\left(h^{\prime}(j)\right)$.

Clearly, (**) holds for $d=0$. Assume that $\left({ }^{*}\right)$ holds for some natural number $d \in$ $\{0, \ldots, r-1\}$ and let $h^{\prime}: d-\operatorname{Sph}\left(\left(N_{i}, \lessdot_{i}, \mu_{i}, \lambda_{i}\right), \gamma_{i}\right) \rightarrow d-\operatorname{Sph}(W, i)$ be a corresponding homomorphism. Let $j_{1}, j_{2} \in N_{i}$ such that $d_{E_{i}}\left(\gamma_{i}, j_{1}\right)=d$ and $d_{E_{i}}\left(\gamma_{i}, j_{2}\right)=d+1$.

Suppose that $j_{1} \lessdot_{i} j_{2}$. As $E_{i}\left[j_{1}\right] \in \rho\left(h^{\prime}\left(j_{1}\right)\right), \rho\left(h^{\prime}\left(j_{1}\right)\right)$ cannot be a final state of $\mathcal{B}_{r}$ so that there is $i_{2} \in[n]$ such that $h^{\prime}\left(j_{1}\right) \lessdot i_{2}$. Clearly, we have $E_{i}\left[j_{2}\right] \in \rho\left(i_{2}\right)$. Analogously, we proceed in the cases $j_{2} \lessdot_{i} j_{1},\left(j_{1}, j_{2}\right) \in \mu_{i}$, and $\left(j_{2}, j_{1}\right) \in \mu_{i}$ to obtain such an element $i_{2}$. Note that $i_{2}$ is uniquely determined by $j_{2}$ and does not depend on the choice of $j_{1}$ or on the specific relation between $j_{1}$ and $j_{2}$. This is less obvious than the corresponding fact in the proof of Claim 4.7 but can be shown along the lines of the following procedure, proving that the extension of the domain of $h^{\prime}$ by elements $j \in N_{i}$ with $d_{E_{i}}\left(\gamma_{i}, j\right)=d+1$ is a homomorphism: 
We show that, for $j, j^{\prime} \in N_{i}$ with $d_{E_{i}}\left(\gamma_{i}, j\right)=d_{E_{i}}\left(\gamma_{i}, j^{\prime}\right)=d+1$, we have $\left(j, j^{\prime}\right) \bigsqcup_{W}^{E_{i}}$ $\left(h^{\prime}(j), h^{\prime}\left(j^{\prime}\right)\right.$ ) (where the elements $h^{\prime}(j)$ and $h^{\prime}\left(j^{\prime}\right)$ are obtained as indicated above). So suppose $j \leftrightarrow_{E_{i}} j^{\prime}$. There are $\ell \in\{0, \ldots, d\}$ and pairwise distinct $j_{0}, \ldots, j_{2(d+1)-\ell} \in N_{i}$, such that

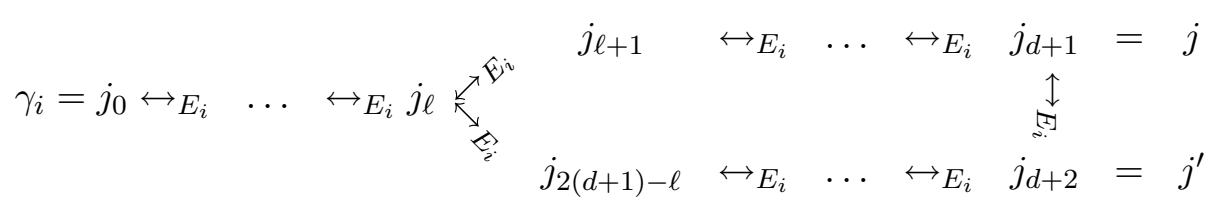

For ease of notation, set $D=2(d+1)-\ell$ and let, for $k \in \mathbb{N}$,

$$
\bmod (k)=\left\{\begin{array}{cc}
k & \text { if } k \leq D \\
((k-\ell) \bmod (D-\ell+1))+\ell & \text { if } k>D
\end{array}\right.
$$

I.e., the mapping mod counts until $D$ and afterwards modulo $D-\ell+1$. According to Claim 4.6, there is a unique infinite sequence $i_{0}, i_{1}, \ldots \in[n]$ such that

- $i_{0}=i$,

- for each $k \in \mathbb{N}, E_{i}\left[j_{\bmod (k)}\right] \in \rho\left(i_{k}\right)$, and

- for each $k \in \mathbb{N},\left(j_{\bmod (k)}, j_{\bmod (k+1)}\right) \sqsubseteq_{W}^{E_{i}}\left(i_{k}, i_{k+1}\right)$.

In what follows, we show that $i_{D+1}=i_{\ell}$, which implies $\left(j_{d+1}, j_{d+2}\right) \sqsubseteq_{W}^{E_{i}}\left(i_{d+1}, i_{d+2}\right)$ so that $\left(j_{d+1}, j_{d+2}\right) \sqsubseteq_{W}^{E_{i}}\left(h^{\prime}\left(j_{d+1}\right), h^{\prime}\left(j_{d+2}\right)\right)$. There is a circular string $w=e_{\ell} \ldots e_{D} \in \Delta^{+}$such that - $j_{\ell} \stackrel{w}{\Longrightarrow} E_{i} j_{\ell}$,

- $j_{\ell} \stackrel{e_{\ell \ldots e_{\ell+k-1}}}{\longrightarrow} E_{i} j_{\ell+k}$ for each $k \in\{1, \ldots, D-\ell\}$, and

- $i_{\ell} \stackrel{w^{k}}{\Longrightarrow} i_{\ell+k(D-\ell+1)}$ for each $k \geq 1$.

We can obtain such a $w$ by setting, for each $k \in\{\ell, \ldots, D\}$,

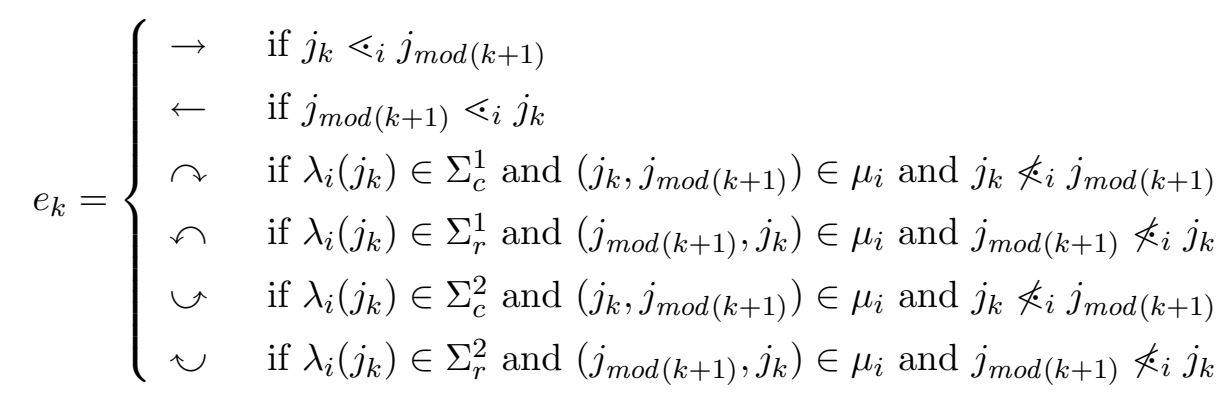

As $[n]$ is a finite set5, there are $p, q \in \mathbb{N}$ such that $\ell \leq p<q$ and $i_{p}=i_{q}$. We choose $p$ and $q$ such that $i_{\ell}, \ldots, i_{q-1}$ are pairwise distinct. We have both $E_{i}\left[j_{\bmod (p)}\right] \in \rho\left(i_{p}\right)$ and $E_{i}\left[j_{\bmod (q)}\right] \in \rho\left(i_{p}\right)$. According to the definition of the set of states of $\mathcal{B}_{r}$, this implies $j_{\bmod (p)}=j_{\bmod (q)}$. Let us distinguish three cases:

Case 1: $p=\ell$ and $q=\ell+k(D-\ell+1)$ for some $k \geq 1$. Then, $i_{\ell} \stackrel{w^{k}}{\longrightarrow} i_{\ell+k(D-\ell+1)}$ so that, according to Proposition 4.5, we have $k=1$ and $i_{\ell}=i_{D+1}$, and we are done.

\footnotetext{
${ }^{5}$ In the context of infinite nested words, this argument can be replaced with the fact that, starting in $i$, there is no infinite sequence of pairwise distinct nodes that follows the infinite sequence of directions $w^{\omega}$, i.e., the infinite repetition of $w$ (see Section [6).
} 
Case 2: $p>\ell$ and $q=p+k(D-\ell+1)$ for some $k \geq 1$. Setting $e=e_{\bmod (p-1)}$, we have both $i_{p-1} \stackrel{e}{\longleftrightarrow} W i_{p}$ and $i_{q-1} \stackrel{e}{\hookrightarrow} W i_{p}$, which is a contradiction, as $i_{p-1} \neq i_{q-1}$.

Case 3: $p \geq \ell$ and $q \neq p+k(D-\ell+1)$ for every $k \geq 1$. But this implies $\bmod (p) \neq \bmod (q)$ and, as the $j_{\ell}, \ldots, j_{D}$ are pairwise distinct, $j_{\bmod (p)} \neq j_{\bmod (q)}$, a contradiction.

This concludes the proof of Claim 4.8.

So let $h: r-\operatorname{Sph}(W, i) \rightarrow \operatorname{core}(\rho(i))$ and $h^{\prime}: \operatorname{core}(\rho(i)) \rightarrow r-\operatorname{Sph}(W, i)$ be the unique homomorphisms that we obtain following the constructive proofs of Claims 4.7 and 4.8, respectively. It is now immediate that $h$ is injective, $h^{-1}=h^{\prime}$, and $h: r$-Sph $(W, i) \rightarrow$ $\operatorname{core}(\rho(i))$ is an isomorphism.

Recall that $\eta: Q \rightarrow$ Spheres $_{r}(\widetilde{\Sigma})$ shall map the empty set to an arbitrary sphere and a nonempty set $\mathcal{E} \in Q$ onto core $(\mathcal{E})$. Indeed, we constructed a generalized 2NWA $\mathcal{B}_{r}=\left(Q, \delta, Q_{I}, F, C\right)$ together with a mapping $\eta: Q \rightarrow$ Spheres $_{r}(\widetilde{\Sigma})$ such that

- $\mathcal{L}\left(\mathcal{B}_{r}\right)$ is the set of all nested words over $\widetilde{\Sigma}$ (cf. Section 4.2.2), and

- for every nested word $W \in \mathbb{N W}(\widetilde{\Sigma})$, every accepting run $\rho$ of $\mathcal{B}_{r}$ on $W$, and every node $i$ of $W$, we have $\eta(\rho(i)) \cong r-\operatorname{Sph}(W, i)$ (cf. Section 4.2.3).

This shows Proposition 4.1.

\section{Grids and Monadic Second-Order Quantifier Alternation}

In this section, we show that the monadic second-order quantifier-alternation hierarchy over nested words is infinite. In other words, the more alternation of second-order quantification we allow, the more expressive formulas become. From this, we can finally deduce that 2 -stack visibly pushdown automata cannot be complemented in general. In the proof, we use results that have been gained in the setting of grids. By means of first-order reductions from grids into nested words, we can indeed transfer expressiveness results for grids to the nested-word setting. Let us first state a general result from [17], starting with the formal definition of a strong first-order reduction:

Definition 5.1 ( [17], Definition 32). Let $\mathcal{C}$ and $\mathcal{C}^{\prime}$ be classes of structures over relational signatures $\tau$ and $\tau^{\prime}$, respectively. A strong first-order reduction from $\mathcal{C}$ to $\mathcal{C}^{\prime}$ with rank $m \geq 1$ is an injective mapping $\Phi: \mathcal{C} \rightarrow \mathcal{C}^{\prime}$ such that the following hold:

(1) For every $G \in \mathcal{C}$, the universe of the structure $\Phi(G)$ is $\bigcup_{k \in\{1, \ldots, m\}}(\{k\} \times \operatorname{dom}(G))$, i.e., the disjoint union of $m$ copies of $\operatorname{dom}(G)$, where $\operatorname{dom}(G)$ shall denote the universe of $G$.

(2) There exists some $\psi\left(x_{1}, \ldots, x_{m}\right) \in \operatorname{FO}\left(\tau^{\prime}\right)$ such that, for every structure $G \in \mathcal{C}$, every $u_{1}, \ldots, u_{m} \in \operatorname{dom}(G)$, and every $k_{1}, \ldots, k_{m} \in[m], \Phi(G) \models \psi\left[\left(k_{1}, u_{1}\right), \ldots,\left(k_{m}, u_{m}\right)\right]$ iff $\left(\left(k_{1}, u_{1}\right), \ldots,\left(k_{m}, u_{m}\right)\right)=\left(\left(1, u_{1}\right), \ldots,\left(m, u_{1}\right)\right)$. (The intuition is that $u \in \operatorname{dom}(G)$ is represented by a model $((1, u), \ldots,(m, u))$ of $\psi$.)

(3) For every relation symbol $r^{\prime}$ from $\tau^{\prime}$, say with arity $l$, and every $\kappa:[l] \rightarrow[m]$, there is $\varphi_{\kappa}^{r^{\prime}}\left(x_{1}, \ldots, x_{l}\right) \in \mathrm{FO}(\tau)$ such that, for each $G \in \mathcal{C}$ and each $u_{1}, \ldots, u_{l} \in \operatorname{dom}(G)$, $G \models \varphi_{\kappa}^{r^{\prime}}\left[u_{1}, \ldots, u_{l}\right]$ iff $\Phi(G) \models r^{\prime}\left[\left(\kappa(1), u_{1}\right), \ldots,\left(\kappa(l), u_{l}\right)\right]$.

(4) For every relation symbol $r$ from $\tau$, say with arity $l$, there is $\varphi^{r}\left(x_{1}, \ldots, x_{l}\right) \in \operatorname{FO}\left(\tau^{\prime}\right)$ such that, for each $G \in \mathcal{C}$ and each $u_{1}, \ldots, u_{l} \in \operatorname{dom}(G), G \models r\left[u_{1}, \ldots, u_{l}\right]$ iff $\Phi(G) \models$ $\varphi^{r}\left[\left(1, u_{1}\right), \ldots,\left(1, u_{l}\right)\right]$. 
Once we have a strong first-order reduction from $\mathcal{C}$ to $\mathcal{C}^{\prime}$, logical definability carries over from $\mathcal{C}$ to $\mathcal{C}^{\prime}$ :

Theorem 5.2 ( [17], Theorem 33). Let $\mathcal{C}$ and $\mathcal{C}^{\prime}$ be classes of structures over relational signatures $\tau$ and $\tau^{\prime}$, respectively. Let $\Phi: \mathcal{C} \rightarrow \mathcal{C}^{\prime}$ be a strong first-order reduction such that $\Phi(\mathcal{C})$ is $\boldsymbol{\Sigma}_{1}\left(\tau^{\prime}\right)$-definable relative to $\mathcal{C}^{\prime}$. Then, for every $\mathcal{L} \subseteq \mathcal{C}$ and $k \geq 1, \mathcal{L}$ is $\boldsymbol{\Sigma}_{k}(\tau)$ definable relative to $\mathcal{C}$ iff $\Phi(\mathcal{L})$ is $\boldsymbol{\Sigma}_{k}\left(\tau^{\prime}\right)$-definable relative to $\mathcal{C}^{\prime}$.

We proceed as follows. We first recall the notion of the class of grids, of which we know that the monadic second-order quantifier-alternation hierarchy is infinite. Then, we give a strong first-order reduction from the class of grids to the class of nested words over a simple 2-stack visibly pushdown alphabet so that we can deduce that the monadic second-order quantifier-alternation hierarchy over nested words is infinite, too. Note that we will add to ordinary grids some particular labeling in terms of $a$ and $b$, which will simplify the upcoming constructions. It is, however, easy to see that well-known results concerning ordinary grids extend to these extended grids (cf. Theorem 5.3 below).

We fix a signature $\tau_{\text {Grids }}=\left\{P_{a}, P_{b}\right.$, succ $_{1}$, succ $\left._{2}\right\}$ with $P_{a}, P_{b}$ unary and succ $_{1}$, succ $_{2}$ binary relation symbols. Let $n, m \geq 1$ be natural numbers. The $(n, m)$-grid is the $\tau_{\text {Grids }}{ }^{-}$ structure $G(n, m)=\left([n] \times[m], \operatorname{succ}_{1}\right.$, succ $\left._{2}, P_{a}, P_{b}\right)$ such that $\operatorname{succ}_{1}=\{((i, j),(i+1, j)) \mid i \in$ $[n-1], j \in[m]\}, \operatorname{succ}_{2}=\{((i, j),(i, j+1)) \mid i \in[n], j \in[m-1]\}, P_{a}=\{(i, j) \in[n] \times[m] \mid j$ is odd $\}$, and $P_{b}=\{(i, j) \in[n] \times[m] \mid j$ is even $\}$. The $(3,4)$-grid is illustrated in Figure 10, By $\mathbb{G}$, we denote the set of all the grids.

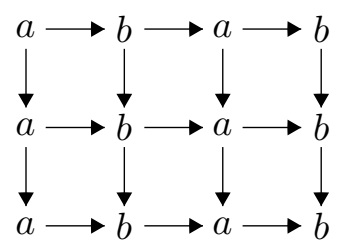

Figure 10: The $(3,4)$-grid

Theorem 5.3 ( [17]). The monadic second-order quantifier-alternation hierarchy over grids is infinite. I.e., for every $k \geq 1$, there is a set of grids that is $\boldsymbol{\Sigma}_{k+1}\left(\tau_{\text {Grids }}\right)$-definable relative to $\mathbb{G}$ but not $\boldsymbol{\Sigma}_{k}\left(\tau_{\text {Grids }}\right)$-definable relative to $\mathbb{G}$.

For the rest of this section, we suppose that $\widetilde{\Sigma}$ is the 2-stack call-return alphabet given by $\Sigma_{c}^{1}=\{a\}, \Sigma_{r}^{1}=\{\bar{a}\}, \Sigma_{c}^{2}=\{b\}, \Sigma_{r}^{2}=\{\bar{b}\}$, and $\Sigma_{\text {int }}=\emptyset$. In particular, the following results assume all alphabets apart from $\Sigma_{\text {int }}$ to be nonempty.

We now describe an encoding $\Phi: \mathbb{G} \rightarrow \mathbb{N W}(\widetilde{\Sigma})$ of grids into nested words over $\widetilde{\Sigma}$. Given $n, m \geq 1$, we let

$$
\Phi(G(n, m)):= \begin{cases}\operatorname{nested}\left(a^{n}\left[(\bar{a} b)^{n}(\bar{b} a)^{n}\right]^{(m-1) / 2} \bar{a}^{n}\right) & \text { if } m \text { is odd } \\ \operatorname{nested}\left(a^{n}\left[(\bar{a} b)^{n}(\bar{b} a)^{n}\right]^{m / 2-1}(\bar{a} b)^{n} \bar{b}^{n}\right) & \text { if } m \text { is even }\end{cases}
$$

The idea is that the first $n$ a's (and, as explained below, the corresponding return events) in a nested word represent the first column of $G(n, m)$ seen from top to bottom; the first $n b$ 's represent the second column, where the column is seen from bottom to top; the second $n a$ 's stand for the third column, again considered from top to bottom, and so on. The encoding 


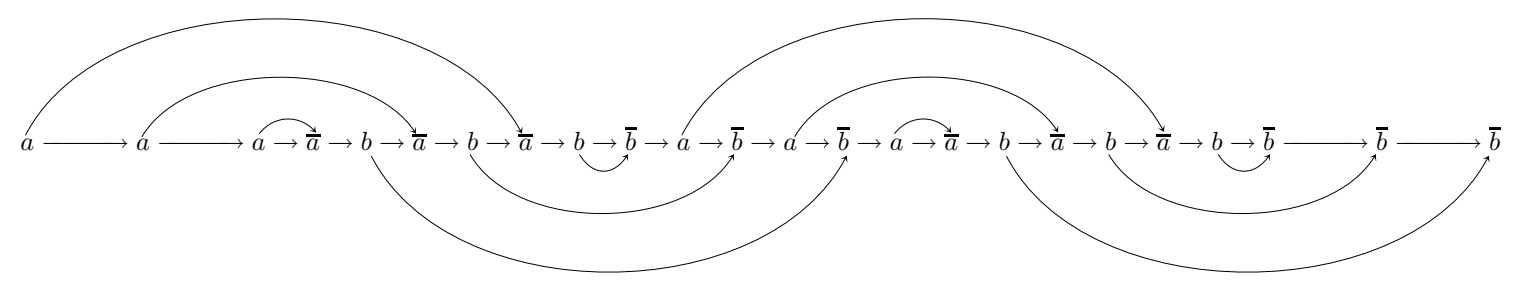

Figure 11: The encoding $\Phi(G(3,4))$ of the $(3,4)$-grid as a nested word

$\Phi(G(3,4))$ of the $(3,4)$-grid as a nested word is depicted in Figure 11. We claim that $\Phi$ is indeed a strong first-order reduction from the set of grids to the set $\mathbb{N} \mathbb{W}(\widetilde{\Sigma})$ of nested words over $\widetilde{\Sigma}$. Observe that $\Phi(G(n, m))$ does not have as domain the set $\{1,2\} \times([n] \times[m])$ as required in the definition of a strong first-order reduction. However, below, we will introduce a bijection $\bar{\chi}_{n, m}:\{1,2\} \times([n] \times[m]) \rightarrow[2 \cdot n \cdot m]$ to identify every element in the domain of $\Phi(G(n, m))$ with some element in $\{1,2\} \times([n] \times[m])$.

Proposition 5.4. We have that $\Phi: \mathbb{G} \rightarrow \mathbb{N W}(\widetilde{\Sigma})$ is a strong first-order reduction with rank 2. Moreover, $\Phi(\mathbb{G})$ is $\boldsymbol{\Sigma}_{1}\left(\tau_{\widetilde{\Sigma}}\right)$-definable relative to $\mathbb{N} \mathbb{W}(\widetilde{\Sigma})$.

Proof. Let us first introduce a useful notation. Given a nested word $W=([n], \lessdot, \mu, \lambda)$ and $c \in \Sigma$ such that $W$ contains at least $k$ positions labeled with $c$, we let $\operatorname{pos}_{c}(W, k)$ denote the least position $i$ in $W$ such that $|\{j \in[i] \mid \lambda(j)=c\}|=k$ (i.e., $\operatorname{pos}_{c}(W, k)$ denotes the position of the $k$-th $c$ in $W)$.

Let $n, m \geq 1$ and let $([2 \cdot n \cdot m], \lessdot, \mu, \lambda)$ refer to $\Phi(G(n, m))$. Recall that $\lambda$ can be seen as the collection of unary relations $\lambda_{c}=\{i \in[2 \cdot n \cdot m] \mid \lambda(i)=c\}$ for $c \in \Sigma$. Let us map any node in the $(n, m)$-grid (i.e., any element from $[n] \times[m])$ to a position of $\Phi(G(n, m)$ ) by defining a function $\chi_{n, m}:[n] \times[m] \rightarrow[2 \cdot n \cdot m]$ as follows:

$$
\chi_{n, m}(i, j)= \begin{cases}\operatorname{pos}_{a}(\Phi(G(n, m)), n \cdot[(j+1) / 2-1]+i) & \text { if } j \text { is odd } \\ \operatorname{pos}_{b}(\Phi(G(n, m)), n \cdot[j / 2-1]+(n+1-i)) & \text { if } j \text { is even }\end{cases}
$$

for any $(i, j) \in[n] \times[m]$. Intuitively, $\chi_{n, m}(i, j) \in[2 \cdot n \cdot m]$ represents the node $(i, j)$ in the $(n, m)$-grid. This mapping is further extended towards a bijection $\bar{\chi}_{n, m}:\{1,2\} \times([n] \times$ $[m]) \rightarrow[2 \cdot n \cdot m]$ as required by Definition 5.1 (item (1)). Namely, we map $\bar{\chi}_{n, m}(1,(i, j))$ onto $\chi_{n, m}(i, j)$ and $\bar{\chi}_{n, m}(2,(i, j))$ onto $\mu\left(\chi_{n, m}(i, j)\right)$.

We are prepared to specify the first-order formulas as supposed in Definition [5.1, Let

$$
\psi\left(x_{1}, x_{2}\right)=\mu\left(x_{1}, x_{2}\right) \text {. }
$$

Indeed, for every $n, m \geq 1, k_{1}, k_{2} \in\{1,2\}$, and $u_{1}, u_{2} \in[n] \times[m]$, we have

$$
\Phi(G(n, m)) \models \psi\left[\bar{\chi}_{n, m}\left(k_{1}, u_{1}\right), \bar{\chi}_{n, m}\left(k_{2}, u_{2}\right)\right] \text { iff }\left(\left(k_{1}, u_{1}\right),\left(k_{2}, u_{2}\right)\right)=\left(\left(1, u_{1}\right),\left(2, u_{1}\right)\right) .
$$

We will identify a map $\kappa:[l] \rightarrow\{1,2\}$ with the tuple $(\kappa(1), \ldots, \kappa(l))$. Let, for $c \in \Sigma$ and $\kappa \in\{1,2\}$,

$$
\varphi_{\kappa}^{\lambda_{c}}(x)=\left\{\begin{array}{cl}
P_{c}(x) & \text { if } c \in\{a, b\} \text { and } \kappa=1 \\
P_{\bar{c}}(x) & \text { if } c \in\{\bar{a}, \bar{b}\} \text { and } \kappa=2 \\
\text { false } & \text { otherwise }
\end{array}\right.
$$


where we let $\overline{\bar{a}}=a$ and $\overline{\bar{b}}=b$. For every $n, m \geq 1, \kappa \in\{1,2\}$, and $u \in[n] \times[m]$, we have

$$
G(n, m) \models \varphi_{\kappa}^{\lambda_{c}}(x)[u] \text { iff } \Phi(G(n, m)) \models(\lambda(x)=c)\left[\bar{\chi}_{n, m}(\kappa, u)\right] .
$$

Further, let, for $\kappa \in\{1,2\} \times\{1,2\}$,

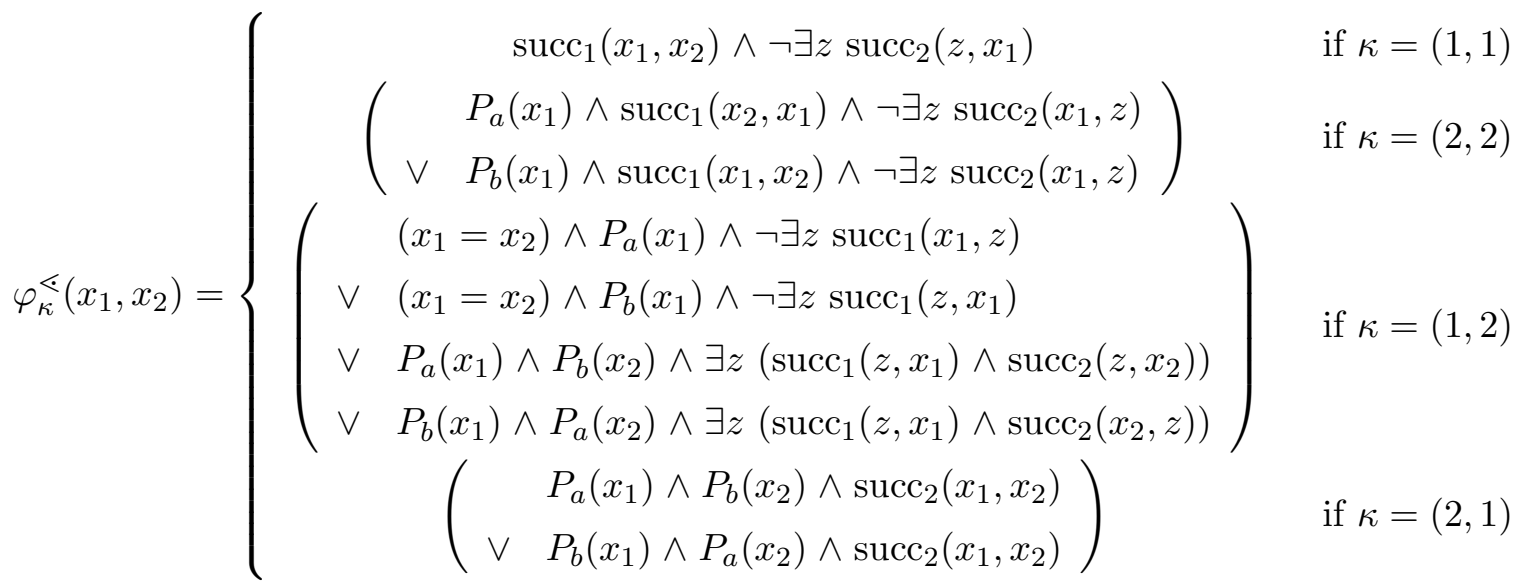

For every $n, m \geq 1, \kappa \in\{1,2\} \times\{1,2\}$, and $u_{1}, u_{2} \in[n] \times[m]$, we have

$G(n, m) \models \varphi_{\kappa}^{\lessdot}\left(x_{1}, x_{2}\right)\left[u_{1}, u_{2}\right]$ iff $\Phi(G(n, m)) \models\left(x_{1} \lessdot x_{2}\right)\left[\bar{\chi}_{n, m}\left(\kappa(1), u_{1}\right), \bar{\chi}_{n, m}\left(\kappa(2), u_{2}\right)\right]$.

Finally, to complete step (3), let, for $\kappa \in\{1,2\} \times\{1,2\}$,

$$
\varphi_{\kappa}^{\mu}\left(x_{1}, x_{2}\right)=\left\{\begin{array}{cl}
x_{1}=x_{2} & \text { if } \kappa=(1,2) \\
\text { false } & \text { otherwise }
\end{array}\right.
$$

Then, for every $n, m \geq 1, \kappa \in\{1,2\} \times\{1,2\}$ and $u_{1}, u_{2} \in[n] \times[m]$,

$$
G(n, m) \models \varphi_{\kappa}^{\mu}\left(x_{1}, x_{2}\right)\left[u_{1}, u_{2}\right] \text { iff } \Phi(G(n, m)) \models\left(\mu\left(x_{1}, x_{2}\right)\right)\left[\bar{\chi}_{n, m}\left(\kappa(1), u_{1}\right), \bar{\chi}_{n, m}\left(\kappa(2), u_{2}\right)\right] \text {. }
$$

Let

$$
\varphi^{P_{a}}(x)=(\lambda(x)=a) \text { and } \varphi^{P_{b}}(x)=(\lambda(x)=b) .
$$

Of course, we have, for each $n, m \geq 1, c \in\{a, b\}$, and $u \in[n] \times[m]$,

$$
G(n, m) \models P_{c}(x)[u] \text { iff } \Phi(G(n, m)) \models\left(\varphi^{P_{c}}\right)\left[\bar{\chi}_{n, m}(1, u)\right] \text {. }
$$

Let

$$
\varphi^{\operatorname{succ}_{1}}\left(x_{1}, x_{2}\right)=\left(\begin{array}{rl}
\lambda\left(x_{1}\right) & =a \wedge \lambda\left(x_{2}\right)=a \wedge\left(x_{1} \lessdot x_{2} \vee \exists z\left(x_{1} \lessdot z \wedge z \lessdot x_{2}\right)\right) \\
\vee & \lambda\left(x_{1}\right)=b \wedge \lambda\left(x_{2}\right)=b \wedge \exists z\left(x_{2} \lessdot z \wedge z \lessdot x_{1}\right)
\end{array}\right)
$$

and let furthermore

$$
\varphi^{\text {succ }_{2}}\left(x_{1}, x_{2}\right)=\exists z\left(\mu\left(x_{1}, z\right) \wedge z \lessdot x_{2}\right) .
$$

Then, for each $n, m \geq 1, u_{1}, u_{2} \in[n] \times[m]$, and $k \in\{1,2\}$, it holds

$$
G(n, m) \models \operatorname{succ}_{k}\left(x_{1}, x_{2}\right)\left[u_{1}, u_{2}\right] \text { iff } \Phi(G(n, m)) \models\left(\varphi^{\mathrm{succ}_{k}}\right)\left[\bar{\chi}_{n, m}\left(1, u_{1}\right), \bar{\chi}_{n, m}\left(1, u_{2}\right)\right] \text {. }
$$

With the above formulas, it is now immediate to verify that $\Phi$ is indeed a strong first-order reduction.

Now observe that $\Phi(\mathbb{G})$ is the "conjunction" of

- the regular expression $\left(a^{+}\left[(\bar{a} b)^{+}(\bar{b} a)^{+}\right]^{*} \bar{a}^{+}\right)+\left(a^{+}\left[(\bar{a} b)^{+}(\bar{b} a)^{+}\right]^{*}(\bar{a} b)^{+} \bar{b}^{+}\right)$,

- the first-order formula $\forall x \exists y(\mu(x, y) \vee \mu(y, x))$, and 
- the first-order property (written in shorthand)

$$
\begin{aligned}
& \forall x_{1}, x_{2}, y_{1}, y_{2}\left(\lambda\left(x_{1}\right)=\lambda\left(x_{2}\right) \wedge \mu\left(x_{1}, y_{1}\right) \wedge \mu\left(x_{2}, y_{2}\right)\right. \\
& \rightarrow \quad\left(\lambda\left(x_{1}\right)=a \wedge x_{2}-x_{1}=1 \rightarrow y_{1}-y_{2} \in\{1,2\}\right) \\
& \wedge\left(\lambda\left(y_{1}\right)=\bar{a} \wedge y_{1}-y_{2}=1 \rightarrow x_{2}-x_{1} \in\{1,2\}\right) \\
& \wedge\left(\lambda\left(y_{1}\right)=\bar{b} \wedge y_{1}-y_{2}=1 \rightarrow x_{2}-x_{1}=2\right) \\
& \wedge\left(x_{2}-x_{1}=2 \wedge \lambda\left(x_{1}+1\right) \neq \lambda\left(x_{1}\right) \rightarrow y_{1}-y_{2} \in\{1,2\}\right) \\
& \left.\wedge\left(y_{1}-y_{2}=2 \wedge \lambda\left(y_{2}+1\right) \neq \lambda\left(y_{2}\right) \rightarrow x_{2}-x_{1} \in\{1,2\}\right)\right)
\end{aligned}
$$

As the regular expression represents a $\boldsymbol{\Sigma}_{1}\left(\tau_{\widetilde{\Sigma}}\right)$-definable property, $\Phi(\mathbb{G})$ is $\boldsymbol{\Sigma}_{1}\left(\tau_{\widetilde{\Sigma}}\right)$-definable relative to $\mathbb{N} \mathbb{W}(\widetilde{\Sigma})$, which concludes the proof of Proposition 5.4 .

Combining Theorem 5.2, Theorem 5.3, and Proposition 5.4, we obtain the following:

Theorem 5.5. The monadic second-order quantifier-alternation hierarchy over nested words is infinite. I.e., for all $k \geq 1$, there is a set of nested words over $\widetilde{\Sigma}$ (with $\widetilde{\Sigma}$ as specified above) that is $\boldsymbol{\Sigma}_{k+1}\left(\tau_{\widetilde{\Sigma}}\right)$-definable relative to $\mathbb{N} \mathbb{W}(\widetilde{\Sigma})$ but not $\boldsymbol{\Sigma}_{k}\left(\tau_{\widetilde{\Sigma}}\right)$-definable relative to $\mathbb{N W}(\widetilde{\Sigma})$.

Recall that Theorem 5.5 relies on a particularly simple call-return alphabet and the presence of at least two stacks. Indeed, its proof is based on the 2-stack call-return alphabet $\widetilde{\Sigma}$, which is given by $\Sigma_{c}^{1}=\{a\}, \Sigma_{r}^{1}=\{\bar{a}\}, \Sigma_{c}^{2}=\{b\}, \Sigma_{r}^{2}=\{\bar{b}\}$, and $\Sigma_{\text {int }}=\emptyset$.

Finally, Theorems 4.3 and 5.5 give rise to the following theorem:

Theorem 5.6. The class of nested-word languages that are recognized by 2VPA is, in general, not closed under complementation. More precisely, there is a set $\mathcal{L}$ of nested words over $\widetilde{\Sigma}$ (with $\widetilde{\Sigma}$ as specified above) such that the following hold:

(1) There is a $2 \mathrm{VPA} \mathcal{A}$ over $\widetilde{\Sigma}$ such that $\mathcal{L}(\mathcal{A})=\mathcal{L}$.

(2) There is no $2 \mathrm{VPA} \mathcal{A}$ over $\widetilde{\Sigma}$ such that $\mathcal{L}(\mathcal{A})=\mathbb{N W}(\widetilde{\Sigma}) \backslash \mathcal{L}$.

This implies that the deterministic model of a $2 \mathrm{VPA}$ (see [13] for its formal definition) is strictly weaker than the general model. This fact was, however, already shown in [13]: Consider the language $L=\left\{(a b)^{m} c^{n} d^{m-n} x^{n} y^{m-n} \mid m \in \mathbb{N}, n \in[m]\right\}$ and the 2-stack callreturn alphabet $\widetilde{\Sigma}$ given by $\Sigma_{c}^{1}=\{a\}, \Sigma_{r}^{1}=\{c, d\}, \Sigma_{c}^{2}=\{b\}, \Sigma_{r}^{2}=\{x, y\}$, and $\Sigma_{\text {int }}=\emptyset$. Then, $L$ is accepted by some 2 VPA over $\widetilde{\Sigma}$ but not by any deterministic 2 VPA over $\widetilde{\Sigma}$. 


\section{Büchi Multi-Stack Visibly Pushdown Automata}

We now transfer some fundamental notions and results from the finite case into the setting of infinite (nested) words.

6.1. Büchi Multi-Stack Visibly Pushdown Automata. Let $K \geq 1$, and let $\widetilde{\Sigma}=$ $\left\langle\left\{\left(\Sigma_{c}^{s}, \Sigma_{r}^{s}\right)\right\}_{s \in[K]}, \Sigma_{\text {int }}\right\rangle$ be a $K$-stack call-return alphabet.

Definition 6.1. A Büchi multi-stack visibly pushdown automaton (Büchi MvPA) over $\widetilde{\Sigma}$ is a tuple $\mathcal{A}=\left(Q, \Gamma, \delta, Q_{I}, F\right)$ whose components agree with those of an ordinary MvPA, i.e., $Q$ is its finite set of states, $Q_{I} \subseteq Q$ is the set of initial states, $F \subseteq Q$ is the set of final states, $\Gamma$ is the finite stack alphabet containing the special symbol $\perp$, and $\delta$ is a triple $\left\langle\delta_{c}, \delta_{r}, \delta_{i n t}\right\rangle$ with $\delta_{c} \subseteq Q \times \Sigma_{c} \times(\Gamma \backslash\{\perp\}) \times Q, \delta_{r} \subseteq Q \times \Sigma_{r} \times \Gamma \times Q$, and $\delta_{\text {int }} \subseteq Q \times \Sigma_{\text {int }} \times Q$.

A Büchi 2-stack visibly pushdown automaton (Büchi 2VPA) is a Büchi MvPA that is defined over a 2-stack alphabet.

Consider an infinite string $w=a_{1} a_{2} \ldots \in \Sigma^{\omega}$. A run of the Büchi MvpA $\mathcal{A}$ on $w$ is a sequence $\rho=\left(q_{0}, \sigma_{0}^{1}, \ldots, \sigma_{0}^{K}\right)\left(q_{1}, \sigma_{1}^{1}, \ldots, \sigma_{1}^{K}\right) \ldots \in\left(Q \times C_{o n t}{ }^{[K]}\right)^{\omega}$ (recall that Cont $=$ $\left.(\Gamma \backslash\{\perp\})^{*} \cdot\{\perp\}\right)$ such that $q_{0} \in Q_{I}, \sigma_{0}^{s}=\perp$ for every stack $s \in[K]$, and [Push], [Pop], and [Internal] as specified in the finite case hold for every $i \in \mathbb{N}_{+}$. We call the run accepting if $\left\{q \mid q=q_{i}\right.$ for infinitely many $\left.i \in \mathbb{N}\right\} \cap F \neq \emptyset$. A string $w \in \Sigma^{\omega}$ is accepted by $\mathcal{A}$ if there is an accepting run of $\mathcal{A}$ on $w$. The such defined (string) language of $\mathcal{A}$ is denoted by $L^{\omega}(\mathcal{A})$.

For the infinite case, we can likewise establish a relational structure of infinite nested words:

Definition 6.2. An infinite nested word over $\widetilde{\Sigma}$ is a structure $\left(\mathbb{N}_{+}, \lessdot, \mu, \lambda\right)$ where $\lessdot=$ $\left\{(i, i+1) \mid i \in \mathbb{N}_{+}\right\}, \lambda: \mathbb{N}_{+} \rightarrow \Sigma$, and $\mu=\bigcup_{s \in[K]} \mu^{s} \subseteq \mathbb{N}_{+} \times \mathbb{N}_{+}$where, for every $s \in[K]$ and $(i, j) \in \mathbb{N}_{+} \times \mathbb{N}_{+},(i, j) \in \mu^{s}$ iff $i<j, \lambda(i) \in \Sigma_{c}^{s}, \lambda(j) \in \Sigma_{r}^{s}$, and $\lambda(i+1) \ldots \lambda(j-1)$ is $s$-well formed.

The set of infinite nested words over $\widetilde{\Sigma}$ is denoted by $\mathbb{N W}^{\omega}(\widetilde{\Sigma})$. Again, given infinite nested words $W=\left(\mathbb{N}_{+}, \lessdot, \mu, \lambda\right)$ and $W^{\prime}=\left(\mathbb{N}_{+}, \lessdot^{\prime}, \mu^{\prime}, \lambda^{\prime}\right), \lambda=\lambda^{\prime}$ implies $W=W^{\prime}$ so that we can represent $W$ as string $(W):=\lambda(1) \lambda(2) \ldots \in \Sigma^{\omega}$. Vice versa, given a string $w \in \Sigma^{\omega}$, there is exactly one infinite nested word $W$ over $\widetilde{\Sigma}$ such that $\operatorname{string}(W)=w$, which we denote $\operatorname{nested}(w)$.

Definition 6.3. A generalized Büchi multi-stack nested-word automaton (generalized Büchi MNwA) over $\widetilde{\Sigma}$ is a tuple $\mathcal{B}=\left(Q, \delta, Q_{I}, F, C\right)$ where $Q, \delta, Q_{I}, F$, and $C$ are as in a generalized MNwA. Recall that, in particular, $\delta$ is a pair $\left\langle\delta_{1}, \delta_{2}\right\rangle$ with $\delta_{1} \subseteq Q \times \Sigma \times Q$ and $\delta_{2} \subseteq Q \times Q \times \Sigma_{r} \times Q$.

We call $\mathcal{B}$ a generalized Büchi 2-stack nested-word automaton (generalized Büchi 2NWA) if it is defined over a 2-stack alphabet.

If $C=\emptyset$, then we call $\mathcal{B}$ a Büchi Mnwa (Büchi 2NwA, if $K=2$ ).

A run of $\mathcal{B}$ on an infinite nested word $W=\left(\mathbb{N}_{+}, \lessdot, \mu, \lambda\right) \in \mathbb{N W}^{\omega}(\widetilde{\Sigma})$ is a mapping $\rho: \mathbb{N}_{+} \rightarrow Q$ such that $(q, \lambda(1), \rho(1)) \in \delta_{1}$ for some $q \in Q_{I}$, and, for all $i \geq 2$, we have

$$
\left\{\begin{aligned}
\left(\rho\left(\mu^{-1}(i)\right), \rho(i-1), \lambda(i), \rho(i)\right) & \in \delta_{2} & & \text { if } \lambda(i) \in \Sigma_{r} \text { and } \mu^{-1}(i) \text { is defined } \\
(\rho(i-1), \lambda(i), \rho(i)) & \in \delta_{1} & & \text { otherwise }
\end{aligned}\right.
$$


The run $\rho$ is accepting if $\rho(i) \in F$ for infinitely many $i \in \mathbb{N}_{+}$and, for all $i \in \mathbb{N}_{+}$with $\rho(i) \in C$, both $\lambda(i) \in \Sigma_{c}$ and $\mu(i)$ is defined. The language of $\mathcal{B}$, denoted by $\mathcal{L}^{\omega}(\mathcal{B})$, is the set of infinite nested words over $\widetilde{\Sigma}$ that allow for an accepting run of $\mathcal{B}$.

As we still have a one-to-one correspondence between strings and nested words, we may let $\mathcal{L}^{\omega}(\mathcal{A})$ with $\mathcal{A}$ a Büchi Mvpa stand for the set $\left\{\operatorname{nested}(w) \mid w \in L^{\omega}(\mathcal{A})\right\}$.

It is now straightforward to adapt Lemma 2.7 and Lemma 2.8 to the infinite setting:

Lemma 6.4. For every generalized Büchi Mnwa $\mathcal{B}$, there is a Büchi MNwA $\mathcal{B}^{\prime}$ such that $\mathcal{L}^{\omega}\left(\mathcal{B}^{\prime}\right)=\mathcal{L}^{\omega}(\mathcal{B})$.

Lemma 6.5. Let $\mathcal{L} \subseteq \mathbb{N W}^{\omega}(\widetilde{\Sigma})$. The following are equivalent:

(1) There is a Büchi Mvpa $\mathcal{A}$ such that $\mathcal{L}^{\omega}(\mathcal{A})=\mathcal{L}$.

(2) There is a Büchi MNwA $\mathcal{B}$ such that $\mathcal{L}^{\omega}(\mathcal{B})=\mathcal{L}$.

6.2. Büchi 2-Stack Visibly Pushdown Automata vs. Logic. In this section, we will again restrict to two stacks. Unfortunately, EMSO logic over infinite nested words turns out to be too weak to capture all the behaviors of Büchi 2VPA. Given that EMSO logic considers a successor relation instead of an order relation, one cannot even express that one particular action occurs infinitely often. To overcome this deficiency, one can introduce a first-order quantifier $\exists^{\infty} x \varphi$ meaning that there are infinitely many positions $x$ to satisfy the property $\varphi[4]$.

So let us fix a 2-stack call-return alphabet $\widetilde{\Sigma}=\left\langle\left\{\left(\Sigma_{c}^{1}, \Sigma_{r}^{1}\right),\left(\Sigma_{c}^{2}, \Sigma_{r}^{2}\right)\right\}, \Sigma_{\text {int }}\right\rangle$ for the rest of the paper. We introduce the logic $\operatorname{MSO}^{\infty}\left(\tau_{\widetilde{\Sigma}}\right)$, which is given by the following grammar:

$$
\begin{aligned}
\varphi::= & \lambda(x)=a|x \lessdot y| \mu(x, y)|x=y| x \in X \mid \\
& \neg \varphi\left|\varphi_{1} \vee \varphi_{2}\right| \exists x \varphi\left|\exists^{\infty} x \varphi\right| \exists X \varphi
\end{aligned}
$$

where $a \in \Sigma$. The fragments $\operatorname{EMSO}^{\infty}\left(\tau_{\widetilde{\Sigma}}\right)$ and $\mathrm{FO}^{\infty}\left(\tau_{\widetilde{\Sigma}}\right)$ are defined as one would expect. The satisfaction relation is as usual concerning the familiar fragment $\operatorname{MSO}\left(\tau_{\widetilde{\Sigma}}\right)$. Moreover, given a formula $\varphi\left(y, x_{1}, \ldots, x_{m}, X_{1}, \ldots, X_{n}\right) \in \operatorname{MSO}^{\infty}\left(\tau_{\widetilde{\Sigma}}\right)$, an infinite nested word $W$, $\left(i_{1}, \ldots, i_{m}\right) \in\left(\mathbb{N}_{+}\right)^{m}$, and $\left(I_{1}, \ldots, I_{n}\right) \in\left(2^{\mathbb{N}_{+}}\right)^{n}$, we set $W \models\left(\exists^{\infty} y \varphi\right)\left[i_{1}, \ldots, i_{m}, I_{1}, \ldots, I_{n}\right]$ iff $W \models \varphi\left[i, i_{1}, \ldots, i_{m}, I_{1}, \ldots, I_{n}\right]$ for infinitely many $i \in \mathbb{N}_{+}$. Given a sentence $\varphi \in$ $\operatorname{MSO}^{\infty}\left(\tau_{\widetilde{\Sigma}}\right)$, we denote by $\mathcal{L}^{\omega}(\varphi)$ the set of infinite nested words over $\widetilde{\Sigma}$ that satisfy $\varphi$.

To establish a connection between the extended logic and our Büchi automata models, we have to provide an extension of Hanf's Theorem.

Theorem 6.6 (cf. [4]). Let $\varphi \in \mathrm{FO}^{\infty}\left(\tau_{\widetilde{\Sigma}}\right)$ be a sentence. There is a positive Boolean combination $\psi$ of formulas of the form

$$
\exists^{=t} x \chi(x) \text { and } \exists^{>t} x \chi(x) \text { and } \exists^{<\infty} x \chi(x) \text { and } \exists^{=\infty} x \chi(x)
$$

where $t \in \mathbb{N}$ and $\chi(x) \in \mathrm{FO}\left(\tau_{\widetilde{\Sigma}}\right)$ is local such that, for every nested word $W \in \mathbb{N W W}^{\omega}(\widetilde{\Sigma})$, we have

$$
W \models \varphi \text { iff } W \models \psi \text {. }
$$

Unfortunately, we do not know if $\psi$ can be computed effectively in this extended setting.

We observe that the 2NWA $\mathcal{B}_{r}$ constructed in the proof of Proposition 4.1 can be easily adapted to obtain its counterpart for infinite nested words:

Proposition 6.7. Let $r \in \mathbb{N}$ be any natural number. There are a generalized Büchi 2NWA $\mathcal{B}_{r}^{\omega}=\left(Q, \delta, Q_{I}, F, C\right)$ over $\widetilde{\Sigma}$ and a mapping $\eta: Q \rightarrow$ Spheres $_{r}(\widetilde{\Sigma})$ such that 
- $\mathcal{L}^{\omega}\left(\mathcal{B}_{r}^{\omega}\right)=\mathbb{N} \mathbb{W}^{\omega}(\widetilde{\Sigma})$ and

- for every $W \in \mathbb{N}^{\omega}(\widetilde{\Sigma})$, every accepting run $\rho$ of $\mathcal{B}_{r}^{\omega}$ on $W$, and every node $i \in \mathbb{N}_{+}$of $W$, we have $\eta(\rho(i)) \cong r-\operatorname{Sph}(W, i)$.

Proof. First, note that Proposition 4.5 and the crucial argument stated in the proof of Claim 4.8 (see Footnote 5) hold for infinite nested words just as well. Now, we look at the generalized 2NWA $\mathcal{B}_{r}=\left(Q, \delta, Q_{I}, F, C\right)$ as constructed in the proof of Proposition 4.1. As the only purpose of the set $F$ of final states is to ensure progress in some states where progress is required in terms of spheres with a non-maximal active node, we can set $\mathcal{B}_{r}^{\omega}$ to be $\left(Q, \delta, Q_{I}, Q, C\right)$, and we are done.

With this, we can easily extend Lemma 4.2 and determine a Büchi 2NWA to detect if a particular sphere occurs infinitely often in an infinite nested word:

Lemma 6.8. Let $r \in \mathbb{N}$ and let $S \in$ Spheres $_{r}(\widetilde{\Sigma})$. There is a generalized Büchi $2 \mathrm{NWA} \mathcal{B}$ over $\widetilde{\Sigma}$ such that $\mathcal{L}^{\omega}(\mathcal{B})=\left\{W \in \mathbb{N W W}^{\omega}(\widetilde{\Sigma}) \mid\right.$ there are infinitely many $i \in \mathbb{N}_{+}$such that $r-\operatorname{Sph}(W, i) \cong S\}$.

Proof. We start from the generalized Büchi 2NwA $\mathcal{B}_{r}^{\omega}=\left(Q, \delta, Q_{I}, Q, C\right)$ and the mapping $\eta: Q \rightarrow$ Spheres $_{r}(\widetilde{\Sigma})$ from Proposition 6.7. To obtain $\mathcal{B}$ as required in the proposition, we simply set the set of final states to be $\{q \in Q \mid \eta(q) \cong S\}$.

Theorem 6.9. Let $\mathcal{L} \subseteq \mathbb{N}^{W}(\widetilde{\Sigma})$ be a set of infinite nested words over the 2-stack callreturn alphabet $\widetilde{\Sigma}$. Then, the following are equivalent:

(1) There is a Büchi 2VPA $\mathcal{A}$ over $\widetilde{\Sigma}$ such that $\mathcal{L}^{\omega}(\mathcal{A})=\mathcal{L}$.

(2) There is a sentence $\varphi \in \operatorname{EMSO}^{\infty}\left(\tau_{\widetilde{\Sigma}}\right)$ such that $\mathcal{L}^{\omega}(\varphi)=\mathcal{L}$.

Proof. To prove $(1) \rightarrow(2)$, one again uses standard methods. Basically, second-order variables $X_{q}$ for $q \in Q$ encode an assignment of states to positions in a nested word. Then, the first-order part of the formula expresses that this assignment is actually an accepting run. To take care of the acceptance condition, we add the disjunction of formulas $\exists^{=\infty} x\left(x \in X_{q}\right)$ with $q$ a final state.

For the direction $(2) \rightarrow(1)$, we make use of Lemmas 6.4, 6.5, 6.8, (a simple variation of) Lemma 4.2, and the easy fact that the class of languages of infinite nested words that are recognized by generalized Büchi 2NWA is closed under union and intersection. With this, the proof proceeds exactly as in the finite case.

\section{Open Problems}

We leave open if visibly pushdown automata still admit a logical characterization in terms of EMSO logic once they are equipped with more than two stacks.

We conjecture that every first-order definable set of nested words over two stacks is recognized by some unambiguous $2 \mathrm{VPA}$, i.e., by a $2 \mathrm{VPA}$ in which an accepting run is unique. To achieve such an automaton, the coloring of spheres as performed by $\mathcal{B}_{r}$ by simply guessing and subsequently verifying it has to be done unambiguously.

We do not know if EMSO logic over nested words becomes more expressive if we allow atomic formulas $x<y$ with the obvious meaning. For this logic, it is no longer possible to apply Hanf's theorem as the degree of the resulting structures is not bounded anymore. 
Our method might lead to logical characterizations for concurrent queue systems, where several visibly pushdown automata communicate with each other via channels [14]. In this extended setting, we deal with both multiple stacks and channels. A corresponding logic then has to provide an additional matching predicate $\operatorname{msg}(x, y)$ to relate the sending and reception of a message (see, for example, [5]). It remains to identify channel architectures for which a logical characterization is possible. Using results from [14], this might lead to partial results concerning the decidability of corresponding satisfiability problems.

Finally, it might be worthwhile to study if our technique leads to a logical characterization of 2VPA for more general 2-stack call-return alphabets as introduced in [8].

Acknowledgment We thank the anonymous referees for their careful reading and many useful remarks.

\section{REFERENCES}

[1] R. Alur and P. Madhusudan. Visibly pushdown languages. In Proceedings of the 36th Annual ACM Symposium on Theory of Computing (STOC 2004), pages 202-211. ACM Press, 2004.

[2] R. Alur and P. Madhusudan. Adding nesting structure to words. In Proceedings of the 10th International Conference on Developments in Language Theory (DLT 2006), volume 4036 of Lecture Notes in Computer Science, pages 1-13. Springer, 2006.

[3] M. F. Atig, B. Bollig, and P. Habermehl. Emptiness of multi-pushdown automata is 2ETIME-complete. In Proceedings of the 12th International Conference on Developments in Language Theory (DLT 2008), volume 5257 of Lecture Notes in Computer Science, pages 121-133. Springer, 2008.

[4] B. Bollig and D. Kuske. Muller message-passing automata and logics. Information and Computation, 206(9-10):1084-1094, 2008.

[5] B. Bollig and M. Leucker. Message-passing automata are expressively equivalent to EMSO logic. Theoretical Computer Science, 358(2-3):150-172, 2006.

[6] L. Breveglieri, A. Cherubini, C. Citrini, and S. Crespi Reghizzi. Multi-push-down languages and grammars. International Journal of Foundations of Computer Science, 7(3):253-292, 1996.

[7] J. Büchi. Weak second order logic and finite automata. Z. Math. Logik Grundlag. Math., 5:66-62, 1960.

[8] D. Carotenuto, A. Murano, and A. Peron. 2-visibly pushdown automata. In Proceedings of the 11th International Conference on Developments in Language Theory (DLT 2007), volume 4588 of Lecture Notes in Computer Science, pages 132-144. Springer, 2007.

[9] M. Droste, P. Gastin, and D. Kuske. Asynchronous cellular automata for pomsets. Theoretical Computer Science, 247(1-2):1-38, 2000.

[10] C. C. Elgot. Decision problems of finite automata design and related arithmetics. Trans. Amer. Math. Soc., 98:21-52, 1961.

[11] W. Hanf. Model-theoretic methods in the study of elementary logic. In J. W. Addison, L. Henkin, and A. Tarski, editors, The Theory of Models. North-Holland, Amsterdam, 1965.

[12] J. E. Hopcroft, R. Motwani, and J. D. Ullman. Introduction to Automata Theory, Languages and Computability. Addison-Wesley, 2000.

[13] S. La Torre, P. Madhusudan, and G. Parlato. A robust class of context-sensitive languages. In Proceedings of the 22nd IEEE Symposium on Logic in Computer Science (LICS 2007), pages 161-170. IEEE Computer Society Press, 2007.

[14] S. La Torre, P. Madhusudan, and G. Parlato. Context-bounded analysis of concurrent queue systems. In Proceedings of the 14th International Conference on Tools and Algorithms for the Construction and Analysis of Systems (TACAS 2008), Lecture Notes in Computer Science, pages 299-314. Springer, 2008.

[15] C. Lautemann, Th. Schwentick, and D. Therien. Logics for context-free languages. In Proceedings of the 1994 Annual Conference of the European Association for Computer Science Logic (CSL 1994), volume 933 of Lecture Notes in Computer Science, pages 205-216, 1995.

[16] L. Libkin. Elements of Finite Model Theory. Springer, 2004.

[17] O. Matz, N. Schweikardt, and W. Thomas. The monadic quantifier alternation hierarchy over grids and graphs. Information and Computation, 179(2):356-383, 2002. 
[18] W. Thomas. Elements of an automata theory over partial orders. In Proceedings of Workshop on Partial Order Methods in Verification (POMIV 1996), volume 29 of DIMACS. AMS, 1996.

[19] W. Thomas. Automata theory on trees and partial orders. In Proceedings of Theory and Practice of Software Development (TAPSOFT 1997), 7th International Joint Conference CAAP/FASE, volume 1214 of Lecture Notes in Computer Science, pages 20-38. Springer, 1997.

[20] W. Thomas. Languages, automata and logic. In A. Salomaa and G. Rozenberg, editors, Handbook of Formal Languages, volume 3, Beyond Words, pages 389-455. Springer, 1997. 